\title{
BMJ Open Urgent care for patients with dementia: a scoping review of associated factors and stakeholder experiences
}

\author{
Jemima Dooley (D) , ${ }^{1}$ Matthew Booker, ${ }^{1}$ Rebecca Barnes, ${ }^{1}$ \\ Penny Xanthopoulou (D) ${ }^{2}$
}

To cite: Dooley J, Booker M, Barnes R, et al. Urgent care for patients with

dementia: a scoping review of associated factors and stakeholder experiences. BMJ Open 2020;10:e037673. doi:10.1136/ bmjopen-2020-037673

- Prepublication history for this paper is available online. To view these files, please visit the journal online (http://dx.doi. org/10.1136/bmjopen-2020037673).

Received 11 February 2020

Revised 22 June 2020

Accepted 08 July 2020
Check for updates

(C) Author(s) (or their employer(s)) 2020. Re-use permitted under CC BY-NC. No commercial re-use. See rights and permissions. Published by BMJ.

${ }^{1}$ Centre for Academic Primary Care, School for Social and Community Medicine, Bristol University, Bristol, UK

${ }^{2}$ Medical School, University of Exeter, Exeter, UK

Correspondence to

Dr Jemima Dooley;

jemima.dooley@bristol.ac.uk

\section{ABSTRACT}

Objectives People with dementia are more vulnerable to complications in urgent health situations due to older age, increased comorbidity, higher dependency on others and cognitive impairment. This review explored the factors associated with urgent care use in dementia and the experiences of people with dementia, informal carers and professionals.

Design Scoping review. The search strategy and data synthesis were informed by people with dementia and carers. Data sources Searches of CINAHL, Embase, Medline, PsycINF0, PubMed were conducted alongside handsearches of relevant journals and the grey literature through 15 January 2019.

Eligibility criteria Empirical studies including all research designs, and other published literature exploring factors associated with urgent care use in prehospital and emergency room settings for people with dementia were included. Two authors independently screened studies for inclusion.

Data extraction and synthesis Data were extracted using charting techniques and findings were synthesised according to content and themes.

Results Of 2967 records identified, 54 studies were included in the review. Specific factors that influenced use of urgent care included: (1) common age-related conditions occurring alongside dementia, (2) dementia as a diagnosis increasing or decreasing urgent care use, (3) informal and professional carers, (4) patient characteristics such as older age or behavioural symptoms and (5) the presence or absence of community support services. Included studies reported three crucial components of urgent care situations: (1) knowledge of the patient and dementia as a condition, (2) inadequate non-emergency health and social care support and (3) informal carer education and stress.

Conclusions The scoping review highlighted a wider variety of sometimes competing factors that were associated with urgent care situations. Improved and increased community support for non-urgent situations, such as integrated care, caregiver education and dementia specialists, will both mitigate avoidable urgent care use and improve the experience of those in crisis.

\section{BACKGROUND}

Over $90 \%$ of people with dementia have another health condition, ${ }^{1}$ with the average number of comorbid conditions of people with dementia aged over 65 double that

\section{Strengths and limitations of this study}

The involvement of people with dementia and informal carers in the protocol design and throughout the review process ensured the research addressed the concerns of all key stakeholders.

- The scoping review methodology allowed a broad research question and inclusion criteria while maintaining a systematic search and data extraction process.

- The primary limitation was the exclusion of research reported in languages other than English.

- Scoping review methodology does not include a formal quality appraisal process, which means the scientific quality of the studies has not been taken into account.

of those without dementia. ${ }^{2}$ This necessitates higher use of healthcare services than people without dementia. ${ }^{3}$ People with dementia are an at-risk patient group when accessing healthcare services in urgent situations due to their cognitive frailty. ${ }^{4}$ They may also be vulnerable to important changes being made when unplanned, urgent care is required, such as introducing or increasing social care support, institutionalisation, prescribing, deprescribing and hospital admission. ${ }^{1}$ However, the impact of dementia on a patient's communication can lead to less involvement in treatment. ${ }^{5}$ Evidence in nonurgent care shows that even when decision making appears shared, people with dementia are not always fully informed of the options and the interests of other stakeholders may take precedent. ${ }^{6} 7$ 'Diagnostic overshadowing' can also occur, where people may be receiving substandard care because dementia takes precedent above other conditions. ${ }^{8}$

Research demonstrates a complex picture when considering how people with dementia access urgent care. A quarter of UK hospital beds are made up with people with dementia over 65 and people with dementia stay in 
hospital for longer than people without. ${ }^{9}$ However, people with dementia are less likely to be taken to hospital after calling the emergency services. ${ }^{10}$ This may indicate that people with dementia are presenting straight to emergency rooms rather than going through ambulatory services. ${ }^{10}$ It also suggests that people with dementia may present through the ambulance service with lower-acuity issues that do not convert to needing hospital attendance.

A further complexity lies in the fact that there are often professional or informal carers involved in urgent care situations in dementia. Informal or professional carers often contact services on behalf of the person with dementia and thus must describe symptoms that are not their own, resulting in complex and inexact triage. ${ }^{11} 12$ Caregivers for those with dementia report a lack of flexibility in community and hospital services to treat dementia symptoms. ${ }^{13}$ There is evidence of informal carer frustration surrounding the lack of alternatives to hospitals and the lack of time in urgent care services to cater for those with complex needs. ${ }^{14}$

While avoidance of hospitalisation is considered increasingly important in dementia care, there is little research that specifically aims to explore what factors affect urgent care situations when patients have dementia or how different stakeholders experience urgent care. ${ }^{8}$ The care provided in urgent situations is broad, encompassing in-hours and out-of-hours primary care, telecare telephone advice services, as well as emergency and prehospital services. ${ }^{15}$ An overview of the literature is, therefore, integral to planning future research in this area. The aim of this scoping review of the literature is to examine: (1) the factors associated with urgent care use in dementia and (2) the experiences of people with dementia, informal carers and professionals in urgent care situations.

\section{METHODS}

A scoping review methodology was chosen due to the broad, multifaceted nature of how people with dementia access urgent care. Scoping reviews enable description of the foci of available research, thus identifying any gaps in the literature. ${ }^{16}$ The Joanna Briggs Institute Methodology for Scoping Reviews was followed ${ }^{17}$; a protocol has been published on the lead author's University website.

\section{Patient and public involvement}

People with dementia and informal carers of those with dementia informed protocol development, ensuring the relevance of the research question to all stakeholders. ${ }^{18}$ This was an iterative process through discussions with the study steering group (one person with dementia and five carers) and through written feedback from five Alzheimer's Society Service User Review Panels. The initial focus of the review was urgent primary care but the group members felt that they would often bypass primary care in urgent situations, instead presenting to emergency services. In response to this stakeholder input, the research questions were revised to include all types of urgent care. The terms 'doctor', 'receptionist', 'choice' and 'choose' were also added to the search strategy. The study steering group of people with experience of dementia and two Alzheimer's Society Living Well with Dementia groups (10 people with dementia) informed the data synthesis by highlighting what features of the coded data were most salient to their experiences. This feedback influenced the synthesis of the findings into the resulting themes.

\section{Research questions}

1. What factors are associated with urgent care use in dementia?

2. What are the experiences of people with dementia, informal carers and professionals in urgent care situations?

\section{Inclusion and exclusion criteria}

Types of studies: Published and unpublished studies were included, with no date restrictions. Only studies published in the English language were included, due to a lack of resources for translation. All study designs, including evidence from qualitative, quantitative and mixedmethods research, were included, as well as theoretical papers and other documents, such as reports, websites, guidelines, leaflets and commentaries. Systematic reviews that met the inclusion criteria were searched and papers relevant to this review were extracted for screening.

Participants: Included studies clearly stated that they include people with dementia, informal carers of people with dementia and/or professional caregivers working with people with dementia. All types and stages of dementia were included, as well as all types of informal or professional carer. Studies exploring older populations or people in care homes where dementia was not specified were not included.

Concepts: Included studies explored urgent care when patients have dementia. Studies that solely reported numbers of people with dementia accessing urgent care as compared with larger cohorts, with no additional contextual findings regarding dementia, were excluded. Similarly, studies that focus on specifically on end-of-life care planning with only incidental discussion of 'do-nothospitalise' orders were not included.

Context: Services accessed in urgent situations were defined as 'non-routine' care, primarily in prehospital settings: urgent primary care, telephone advice services and ambulance services. While studies examining accident and emergency department settings were included as an exploration of the interface between community and hospital care, studies that focused solely of the provision of emergency treatment of acutely life-threatening conditions within hospitals were excluded. Studies that reported hospitalisation as a result of urgent care decision making were included, but trials and reports of interventions where the primary outcome was avoiding hospitalisation were excluded. There is an ongoing systematic 


\begin{tabular}{|c|c|c|}
\hline Databases & Handsearch & Grey literature \\
\hline CINAHL & Aging and Mental Health & Canada Theses \\
\hline Embase & $\begin{array}{l}\text { Alzheimer's Disease and } \\
\text { Associated Disorders }\end{array}$ & $\begin{array}{l}\text { DART European } \\
\text { Theses }\end{array}$ \\
\hline Medline & $\begin{array}{l}\text { American Journal of } \\
\text { Alzheimer's Disease and } \\
\text { other dementias }\end{array}$ & EThOS UK Theses \\
\hline PsycINFO & BMC Geriatrics & $\begin{array}{l}\text { Informa Healthcare } \\
\text { website }\end{array}$ \\
\hline \multirow[t]{10}{*}{ PubMed } & $\begin{array}{l}\text { British Journal of General } \\
\text { Practice }\end{array}$ & $\begin{array}{l}\text { ProQuest: } \\
\text { (wire feeds, } \\
\text { trade journals, } \\
\text { dissertations, } \\
\text { theses, news/ } \\
\text { magazines, } \\
\text { conference papers, } \\
\text { books, reports) }\end{array}$ \\
\hline & Clinical Gerontologist & $\begin{array}{l}\text { WorldCat global } \\
\text { library }\end{array}$ \\
\hline & Dementia & \\
\hline & $\begin{array}{l}\text { International Journal of } \\
\text { Geriatric Psychiatry }\end{array}$ & $\begin{array}{l}\text { Online guidance: } \\
\text { RCGP clinician } \\
\text { training resources, } \\
\text { Alzheimer's } \\
\text { Society website, } \\
\text { Dementia Roadmap } \\
\text { website, Dementia } \\
\text { Knowledge Centre }\end{array}$ \\
\hline & $\begin{array}{l}\text { International } \\
\text { Psychogeriatrics }\end{array}$ & \\
\hline & Journal of Aging Studies & \\
\hline & $\begin{array}{l}\text { Journal of the American } \\
\text { Geriatrics Society }\end{array}$ & \\
\hline & $\begin{array}{l}\text { Academic Emergency } \\
\text { Medicine }\end{array}$ & \\
\hline & Age \& Ageing & \\
\hline & $\begin{array}{l}\text { Archives of Internal } \\
\text { Medicine }\end{array}$ & \\
\hline
\end{tabular}

review of intervention studies aiming to avoid hospitalisation in dementia. ${ }^{19}$

\section{Search strategy}

The information sources and search terms are outlined in table 1 and box 1 . Sources were searched from inception to $15 \mathrm{t}^{\mathrm{h}}$ January 2019. Search terms were chosen through discussion with clinicians, people with dementia and informal carers, and an information specialist.

The search results were downloaded into Endnote X9 and were initially screened for inclusion based on title and abstract, followed by full-text screening. Over a process of three meetings, papers were jointly screened by authors JD and PX, to ensure clarity in the inclusion criteria and to identify any disagreements. JD then independently screened the remaining studies, with PX double screening a further $20 \%$. Where JD was uncertain

\section{Box 1 Search terms}

\section{Search Termsterms}

1. One exp Dementia/

2. (dementia* or alzheimer*).ti,ab,kf,hw.

3. 1 or 2

4. Four exp Ambulatory Care/

5. ((urgent or primary) adj (healthcare or care)).ti,ab,kf,hw.

6. Emergencies/

7. Seven exp Emergency Medical Services/

8. (emergency or emergencies or hotline* or hot line* or telephone or telecare or "111" or "999" or weekend or hospital* or evening* or out of hours or urgent or night* or A\&E or ambulance or ambulatory).ti,ab,kf,hw.

9. 4 or 5 or 6 or 7 or 8

10. Ten exp Health Personnel/

11. (nurse* or nursing staff or doctor* or physician* or paramedic* or receptionist* or care home staff or care home staff or $\mathrm{GP}^{\star}$ or general practitioner*).ti,ab,kf,hw.

12. 10 or 11

13. Decision Making/

14. Health Communication/

15. (decision* or choice* or choos* or response* or understand* or options or triag*).ti,ab,kf,hw.

16. (communicat* adj3 (patient* ${ }^{\star}$ or carer $\left.\left.{ }^{\star}\right)\right) . t i, a b, k f, h w$.

17. 13 or 14 or 15 or 16

18. 3 and 9 and 12 and 17

19. 3 and 9 and 12

over inclusion, studies were discussed as a study team to ensure no relevant materials were excluded.

Reference lists of included papers that were not empirical research (eg, reviews, editorials, guidelines) were searched for any relevant primary research to be included in the final review. Forward and backward citation searches of included papers were then undertaken. Keywords of included papers were then screened against the original search terms to ensure that they had all been included.

\section{Data extraction}

Following scoping review methodology, data were extracted by charting study findings, resulting in a descriptive summary of included studies. JD led on data extraction, with PX independently extracting data from $33 \%$ of the studies to check for accuracy.

\section{Data synthesis}

Study results relevant to the research questions were coded line by line for content, setting and type of participants using NVivo V.12. The codes were grouped according to setting and type of participants to allow description of the studies. They were then regrouped according to content of the codes, using methods from thematic analysis, first creating categories of codes with similar content and then forming groups of categories according to similarities and differences. These were discussed with the patient and public involvement groups to ensure clarity and relevance. This allowed a thematic description of (1) factors 


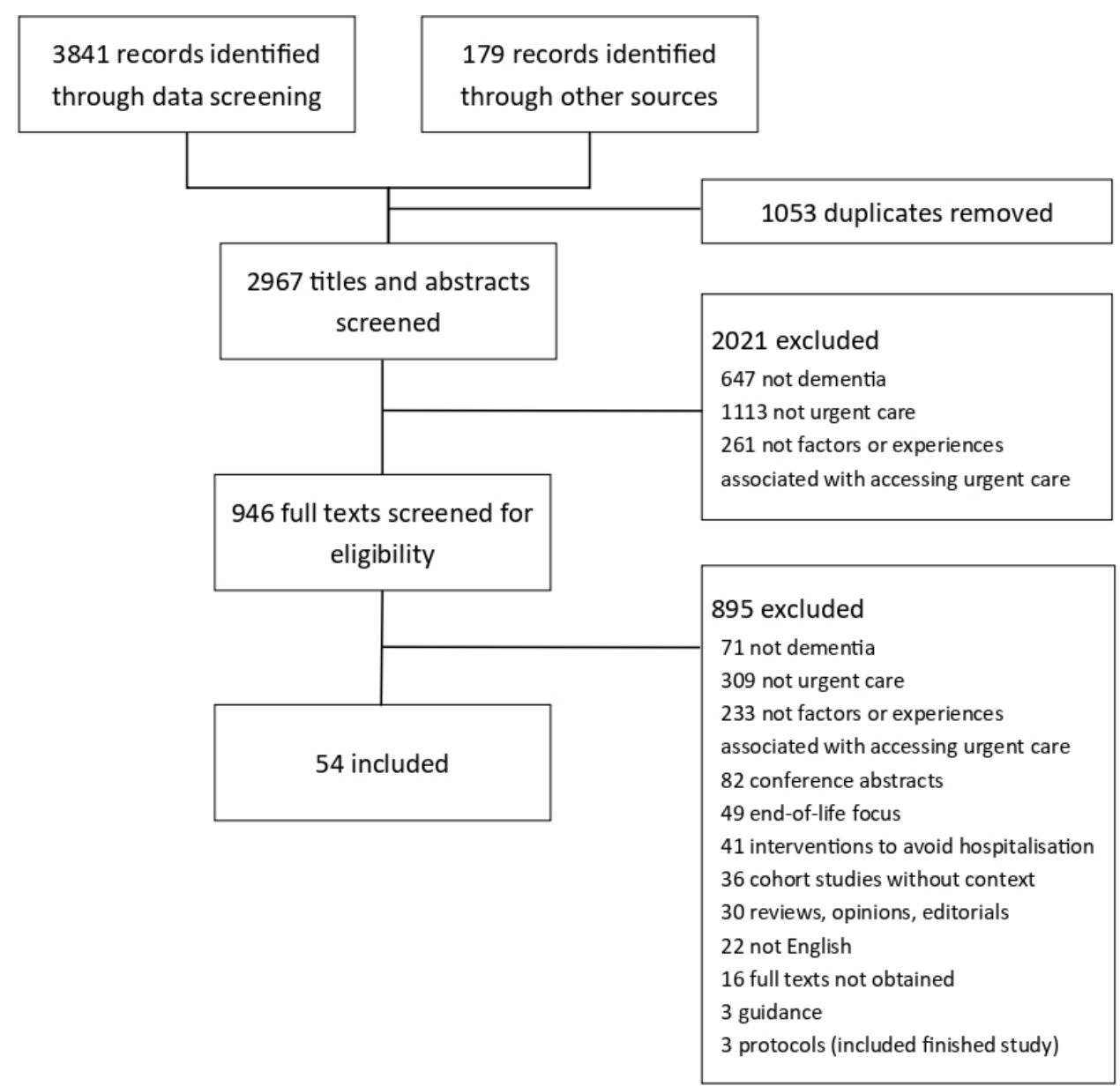

Figure 1 PRISMA flow chart. PRISMA, Preferred Reporting Items for Systematic Reviews and Meta-Analyses.

associated with urgent care use in dementia and (2) stakeholder experiences of urgent care use in dementia.

\section{RESULTS}

\section{Included studies}

Figure 1 contains a flow chart of the screening process. Database searches identified 3841 records, with a further 179 identified from other sources. After duplicates were removed, 2967 titles and abstracts were screened, 946 records were retained for full-text screening. Fifty-four studies were included in the scoping review.

\section{Study characteristics}

Table 2 provides a summary of the included studies. Twenty-six $(48 \%)$ of the studies were conducted in the USA, $10(18.5 \%)$ in the UK, $5(9.5 \%)$ in Canada, 3 in the Netherlands, 2 (1.6\% each) in France and China, and the remaining 5 in Taiwan, Ireland, Republic of Korea, Australia and Italy. The studies were published between 1991 and 2019, with 57\% ( $\mathrm{n}=31)$ published in the last 5 years. Twenty-two $(41 \%)$ of the studies followed a prospective study design. Twelve $(22 \%)$ were qualitative studies, nine $(17 \%)$ were retrospective, five $(9 \%)$ were surveys, five $(9 \%)$ were mixed methods and one followed a Delphi process. The majority of studies included people with all types of dementia or did not specify type of dementia. Four studies included only people with Alzheimer's disease and one study specified inclusion of participants with Alzheimer's disease, Vascular dementia and Parkinson's dementia.

\section{Factors associated with urgent care use in dementia}

Forty-five studies reported factors that were associated with urgent care use in dementia. These are listed in table 3 , illustrating a number of contradictory findings.

\section{Common comorbidities alongside dementia}

Table 4 provides a summary of conditions discussed in the included studies occurring alongside dementia. Comorbidities were mostly described as 'age related', ${ }^{20}$ rather than dementia related, but studies considered whether people with dementia were more likely to access urgent care for these conditions. Two studies reported people with dementia being more susceptible to hospitalisation for 'ambulatory care sensitive' conditions, conditions that could be treated outside of hospital, meaning hospital could have been avoided. ${ }^{21}{ }^{22}$ Nine studies reported a statistically significant increase in urgent service use when people with dementia have other medical conditions. ${ }^{10} 2023-29$ However, there was not a consensus; two 

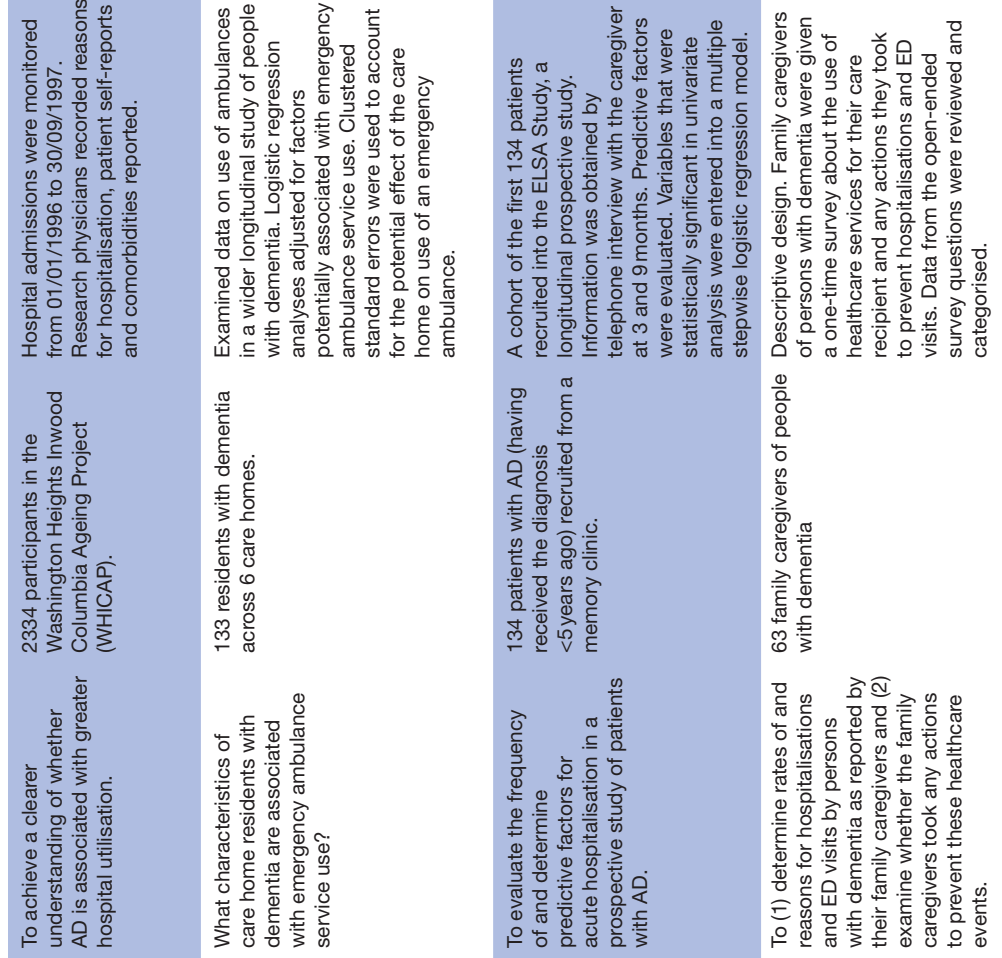

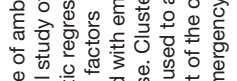

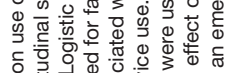
贾

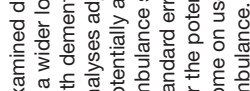

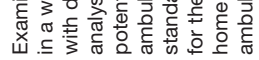
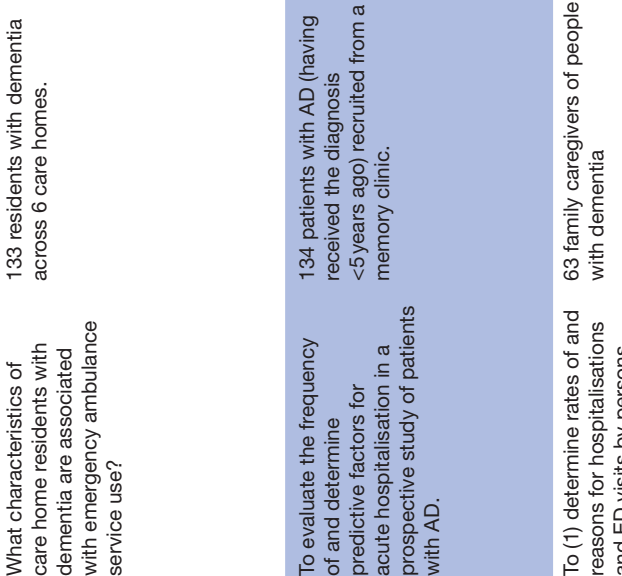

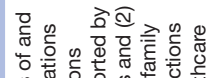

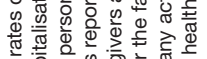

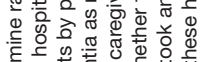

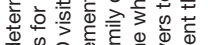

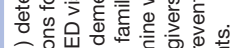

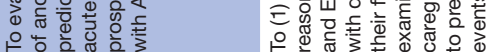
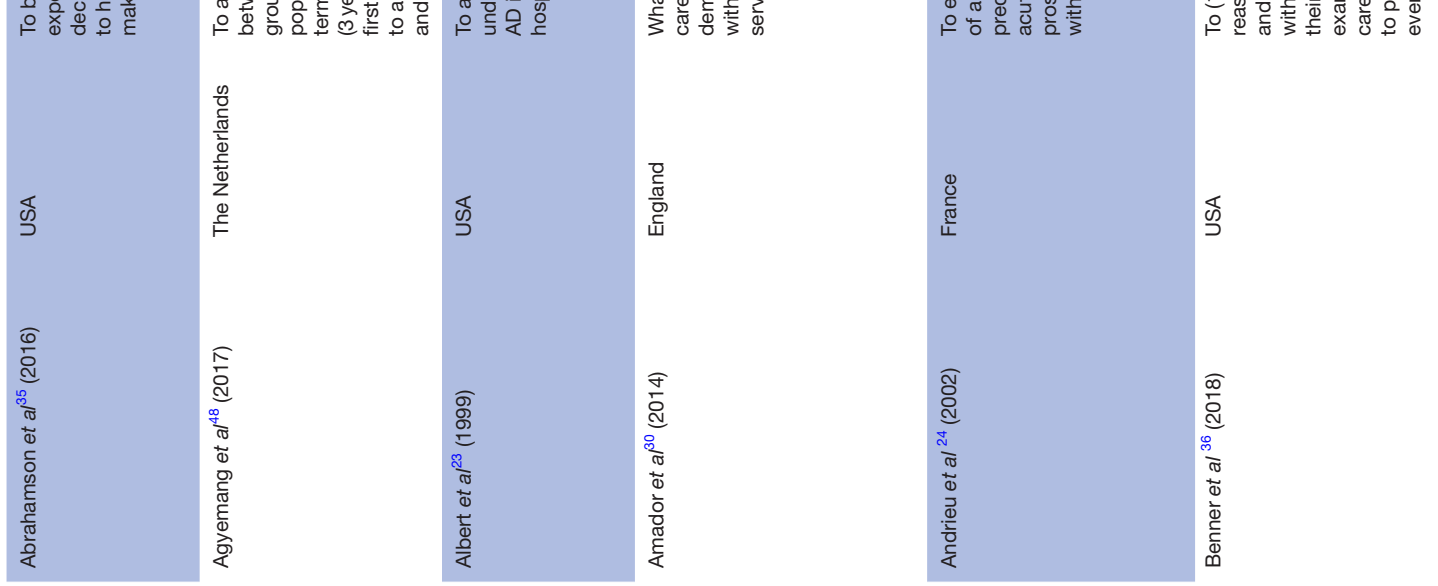


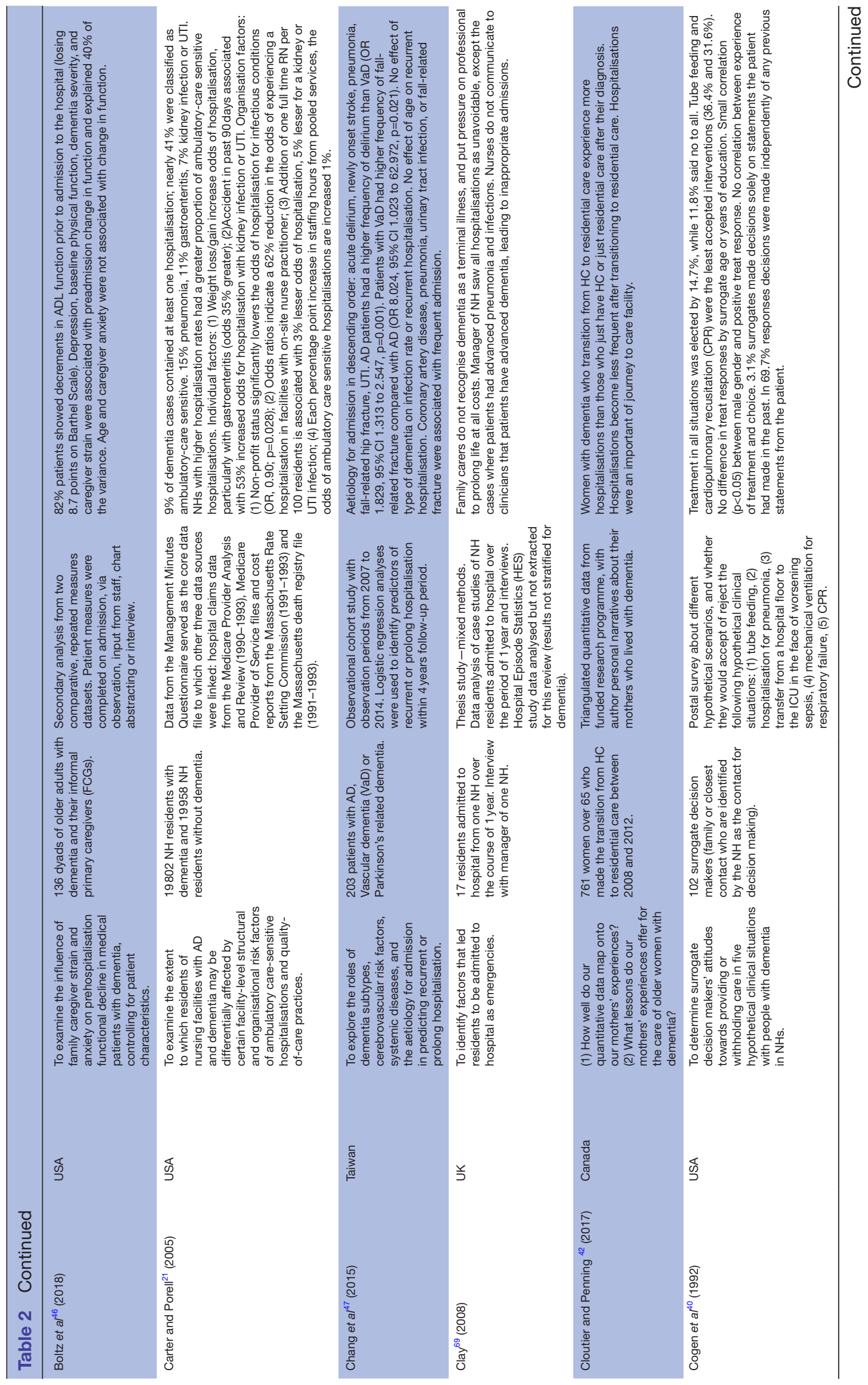




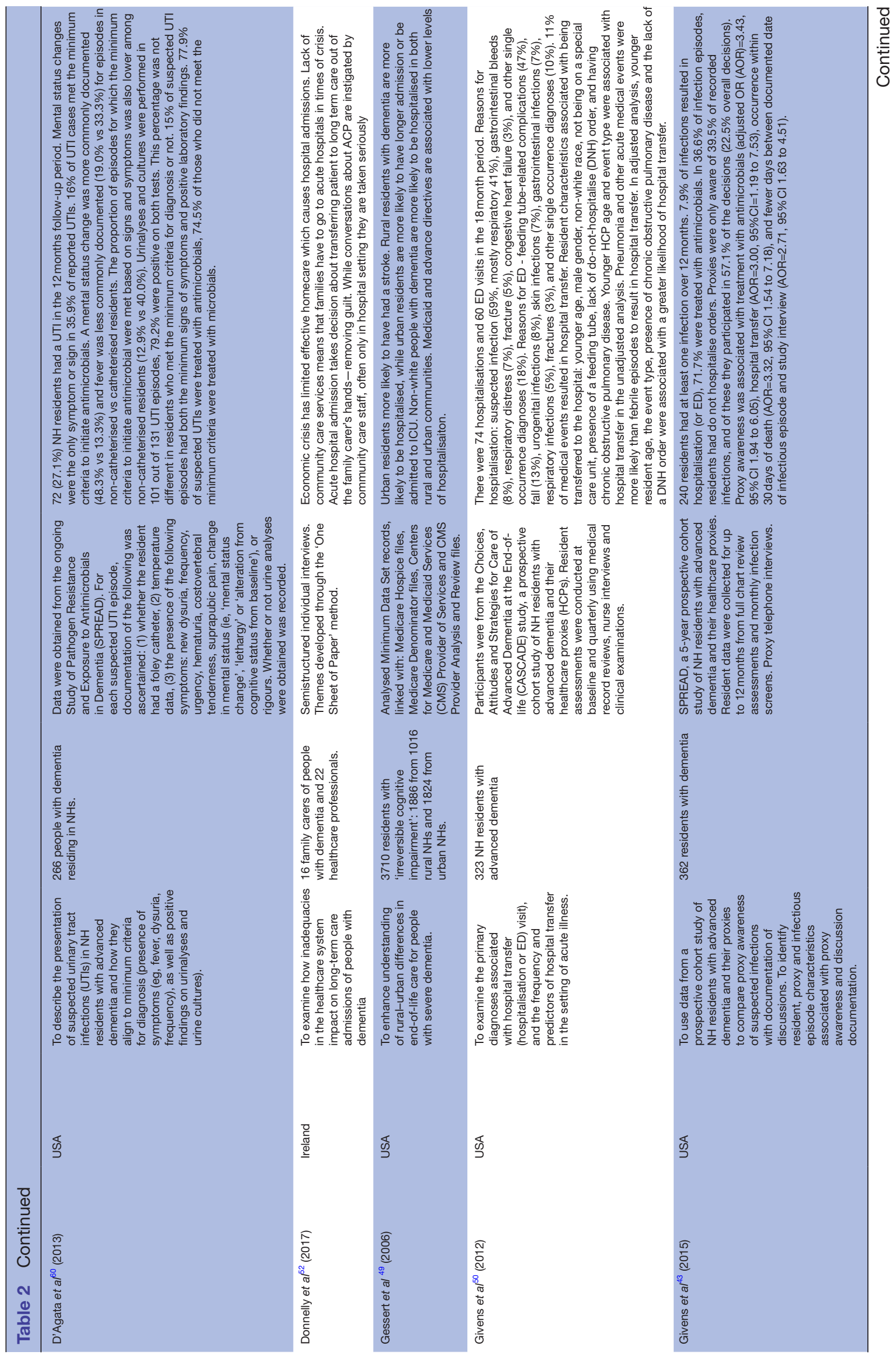




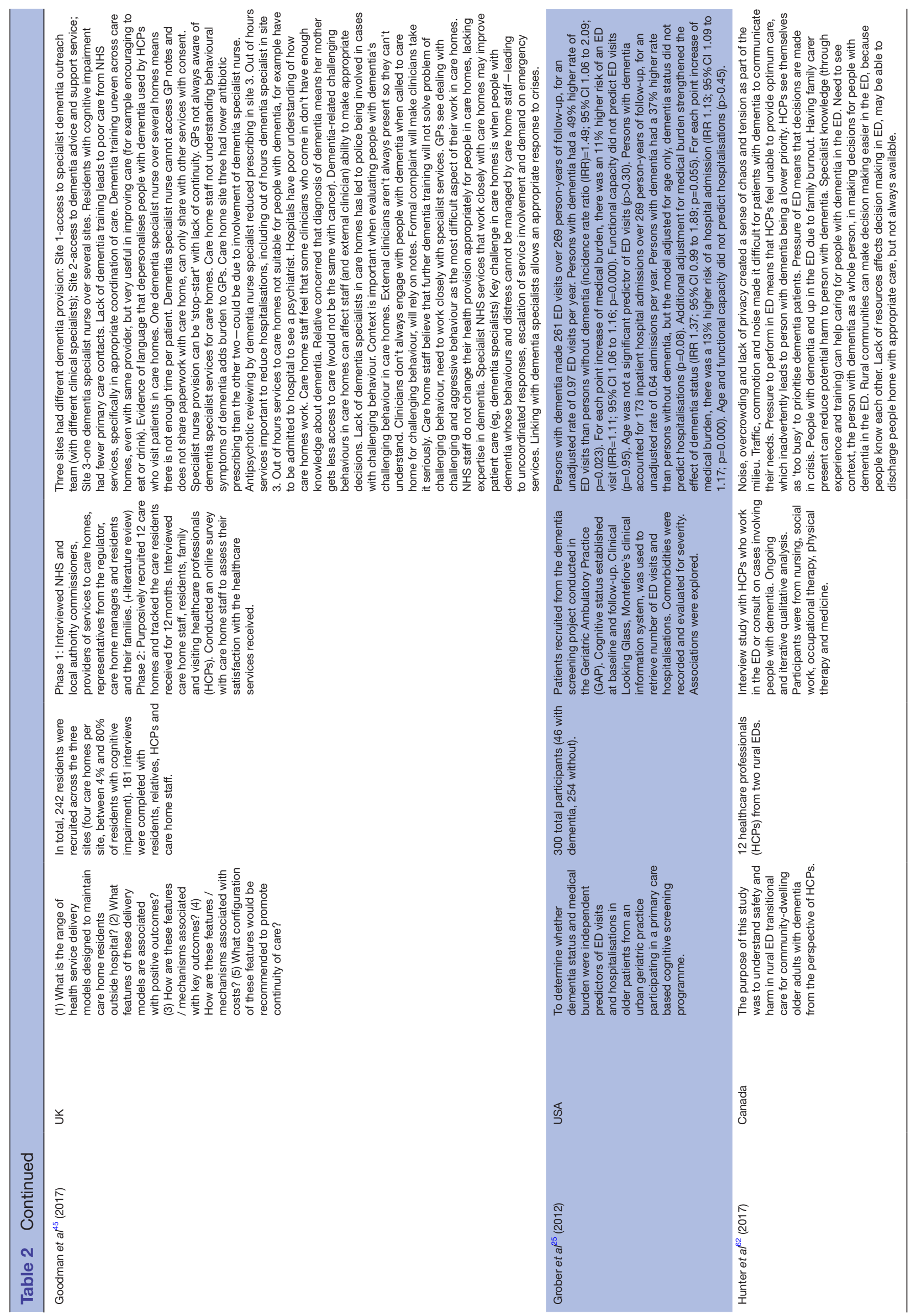

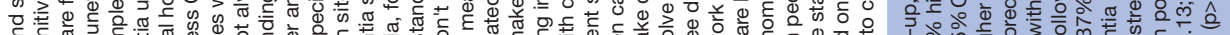

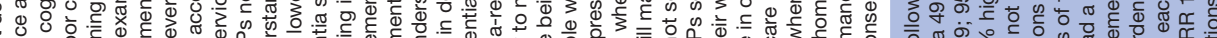

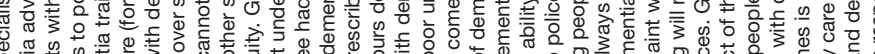

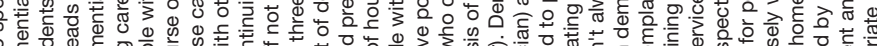

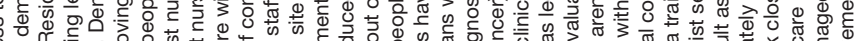

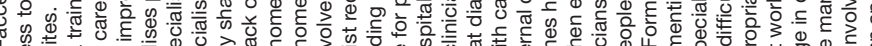

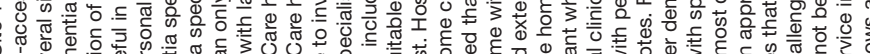

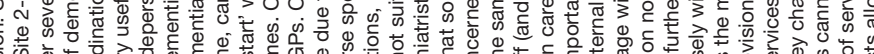

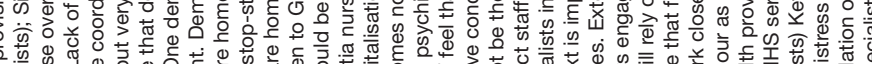

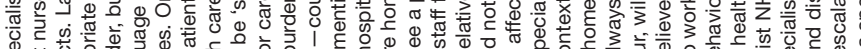

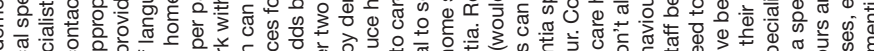

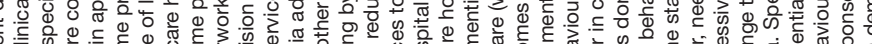

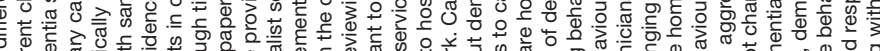

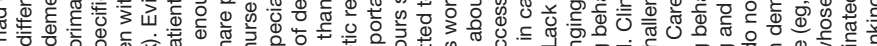

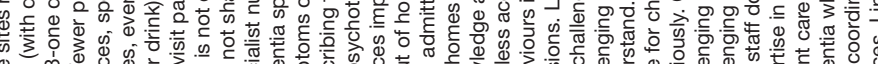

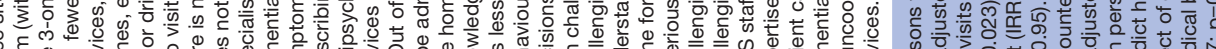

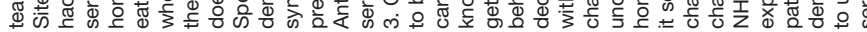

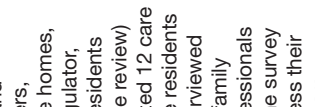

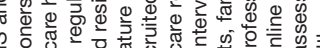

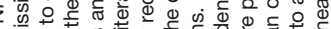

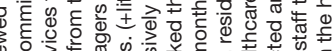

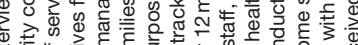

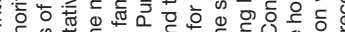

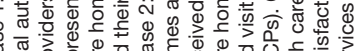

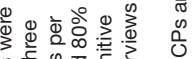

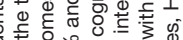

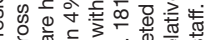

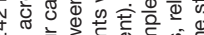

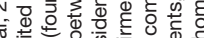

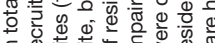

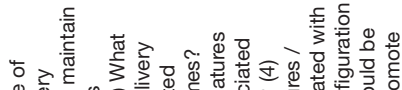

等

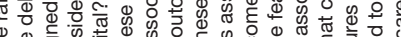
等

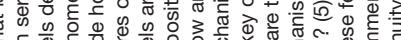
Whin 


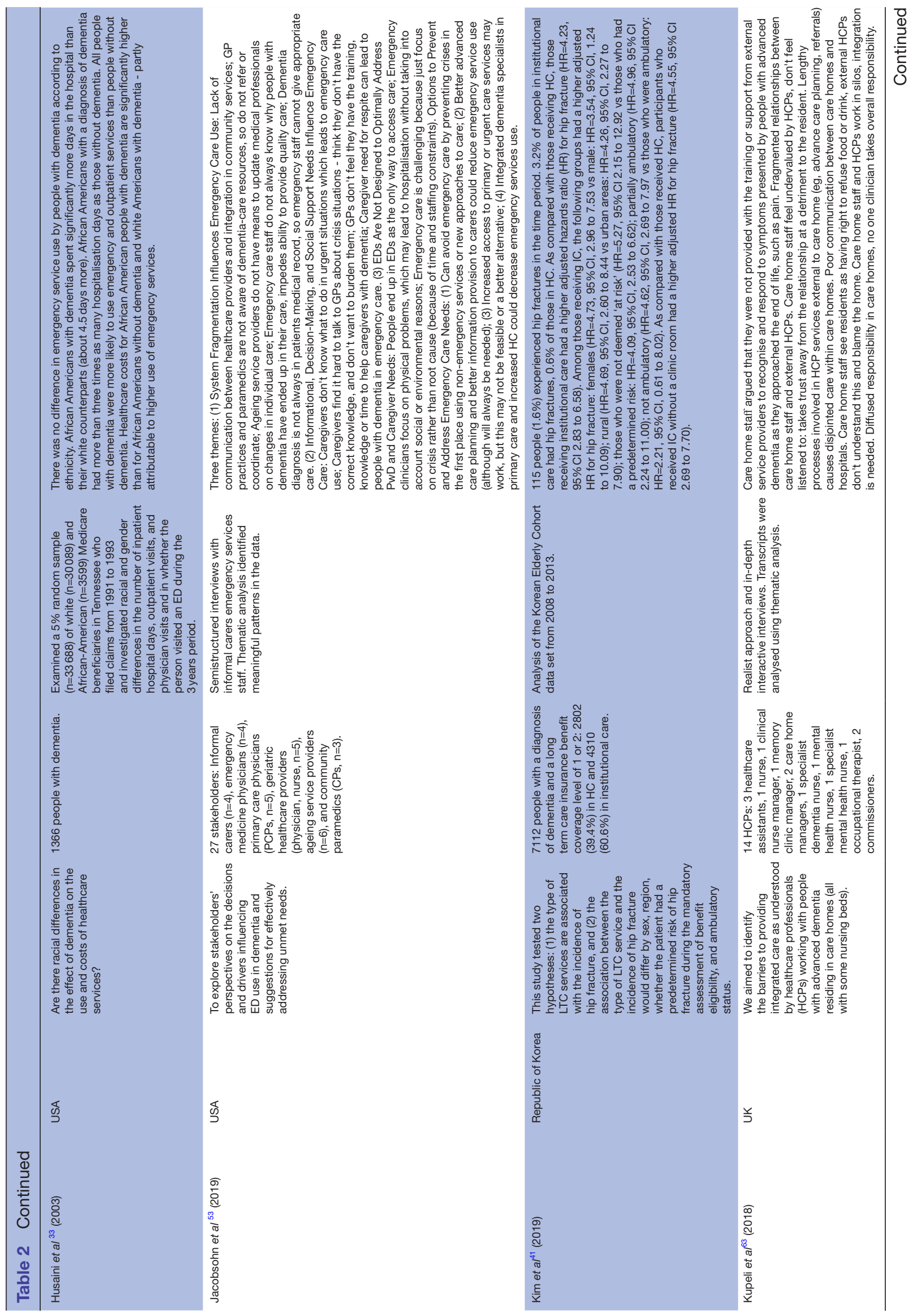




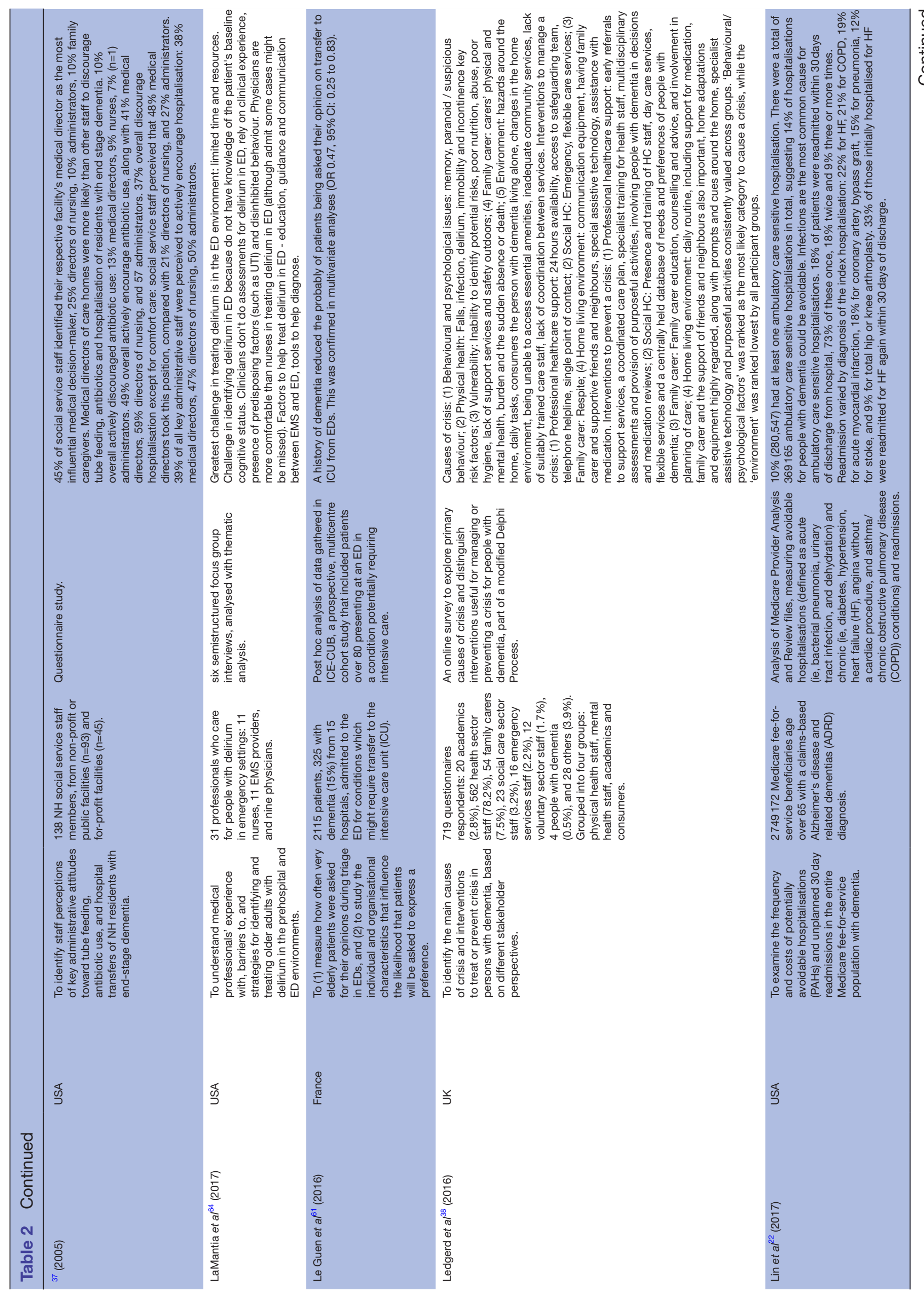




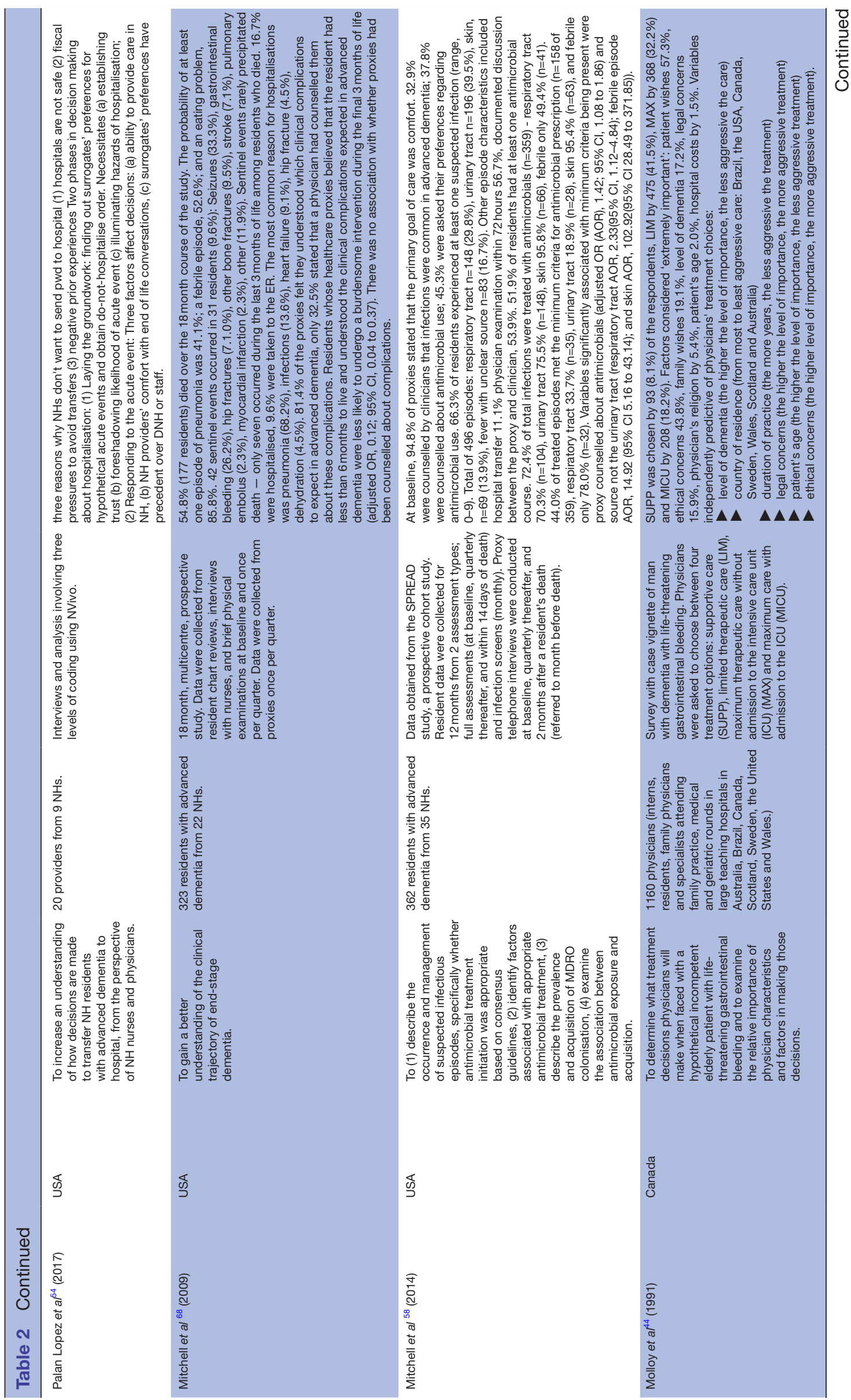




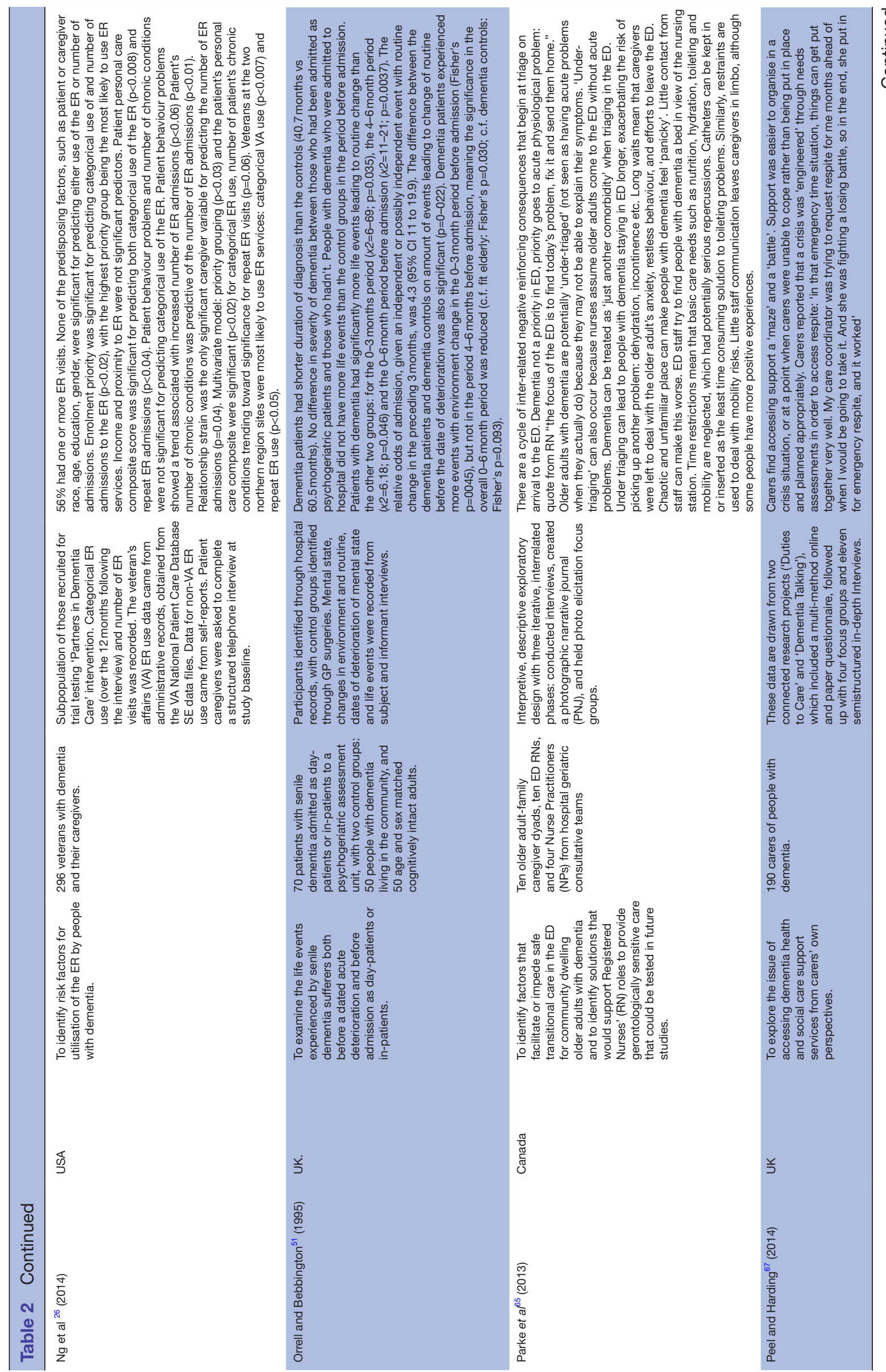




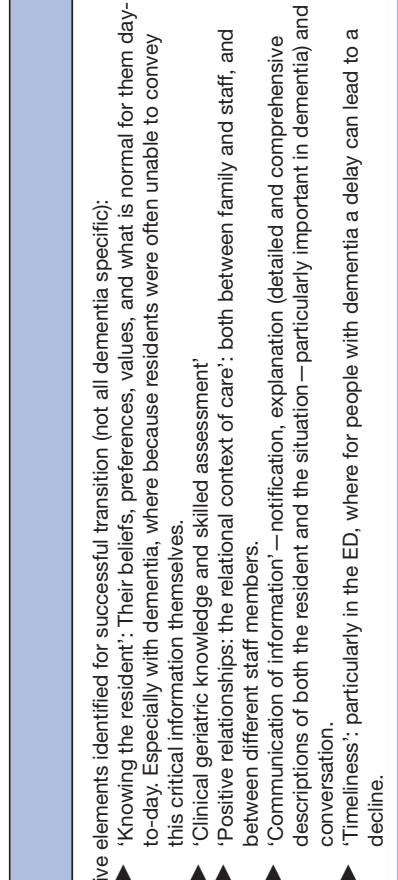

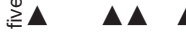

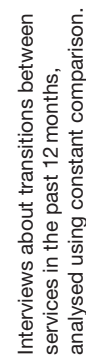

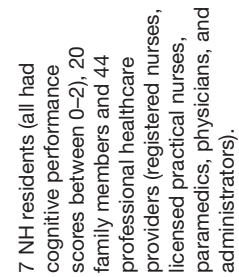

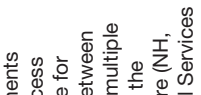

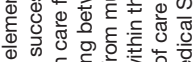

ब.

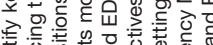

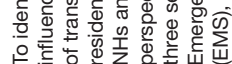

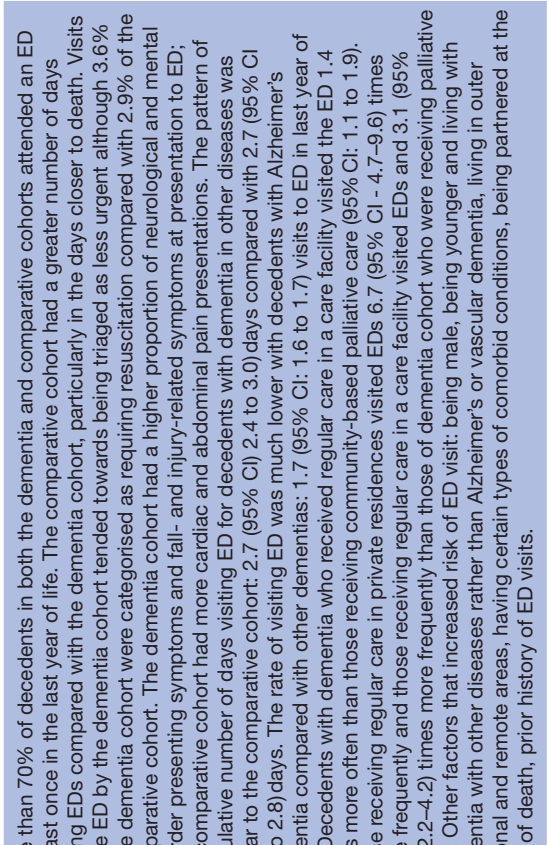

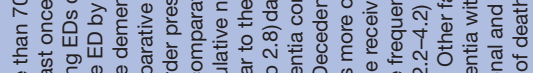
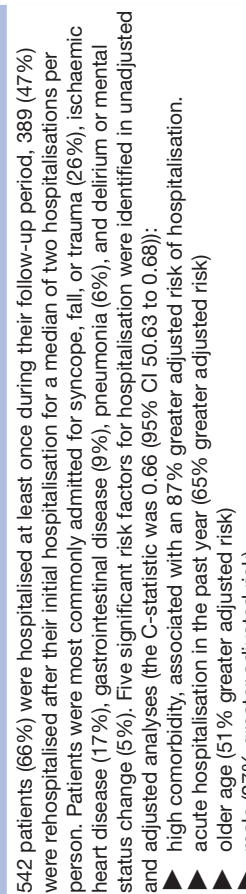

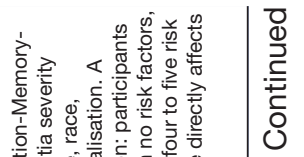

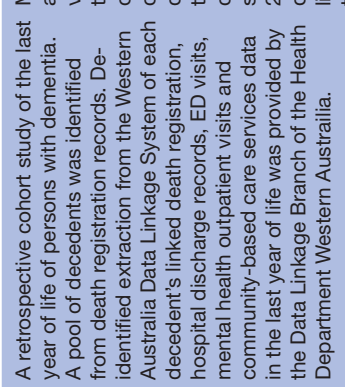

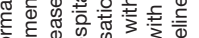

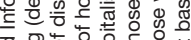

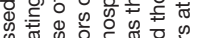

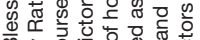

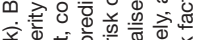

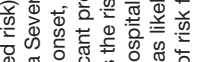

ब.

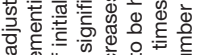

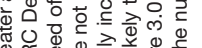

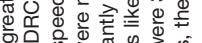

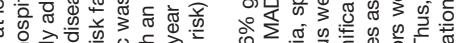

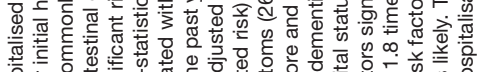

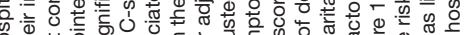

等

等

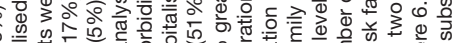

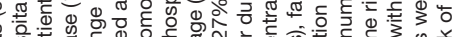

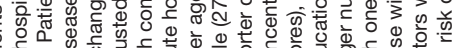

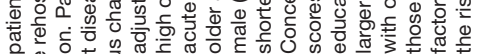

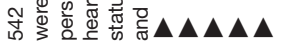
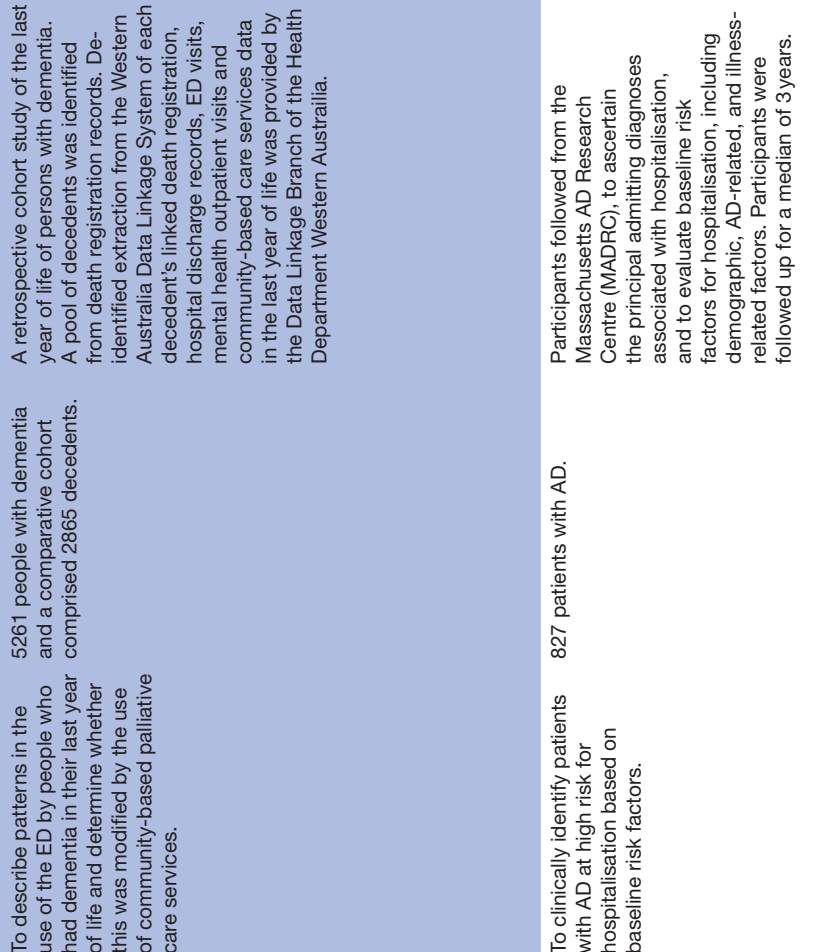

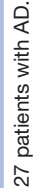

禹
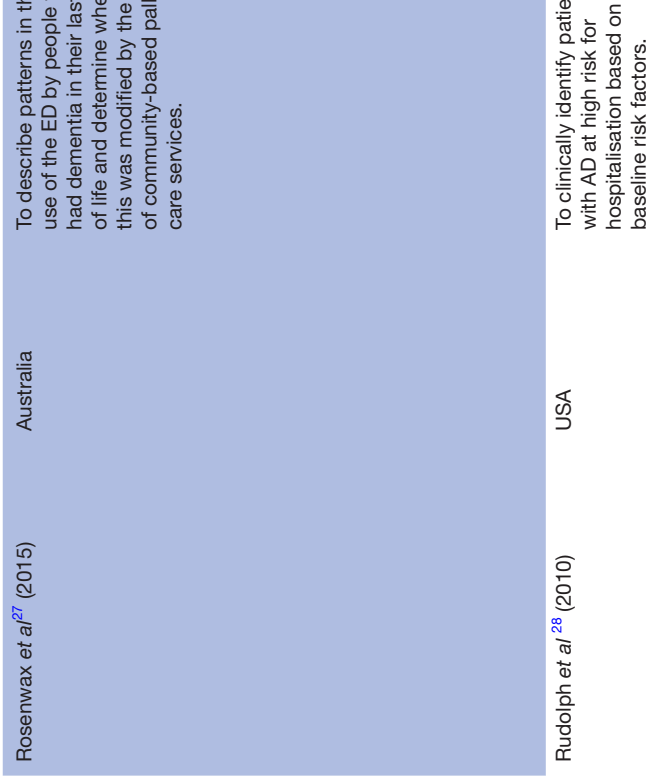


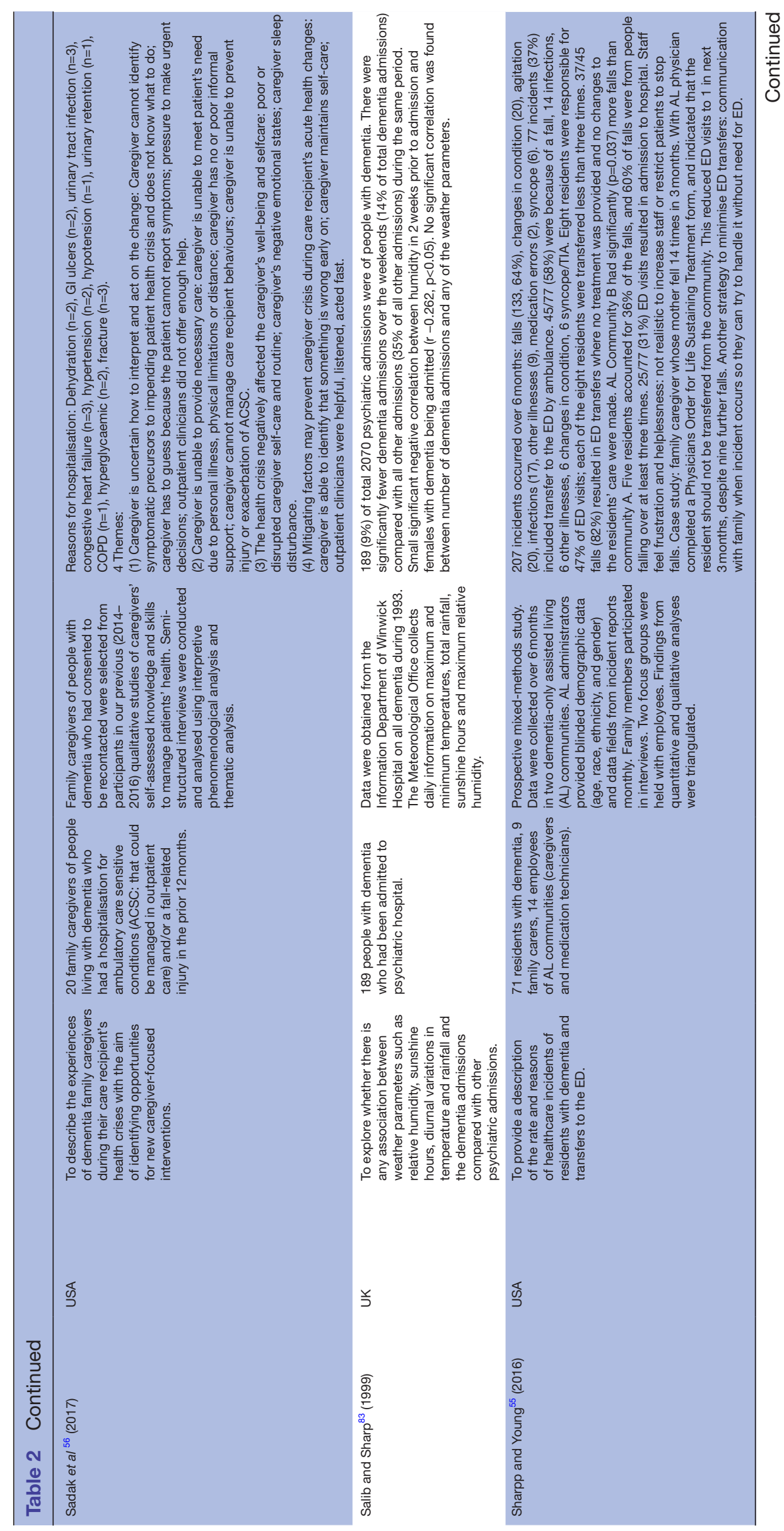




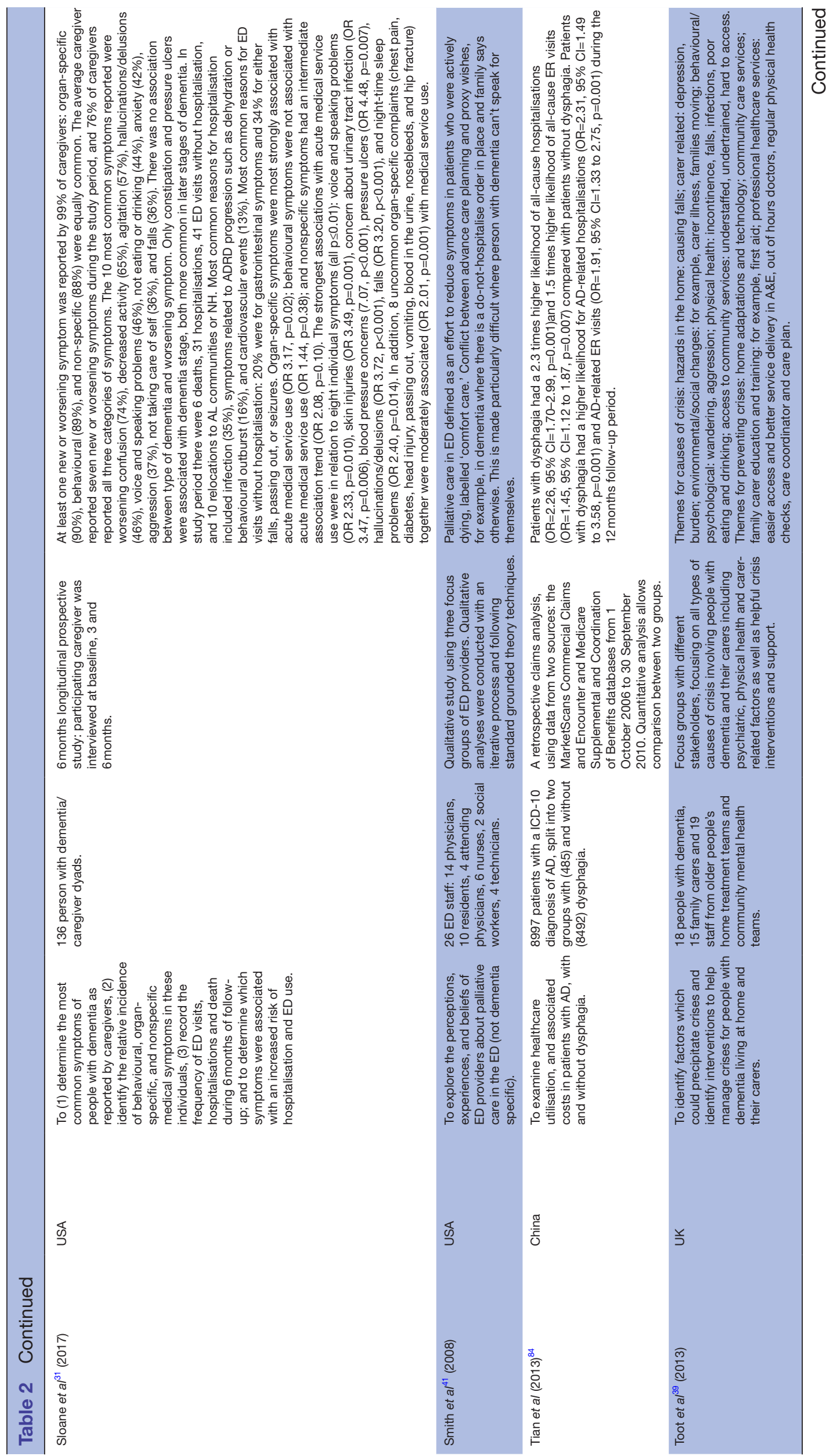




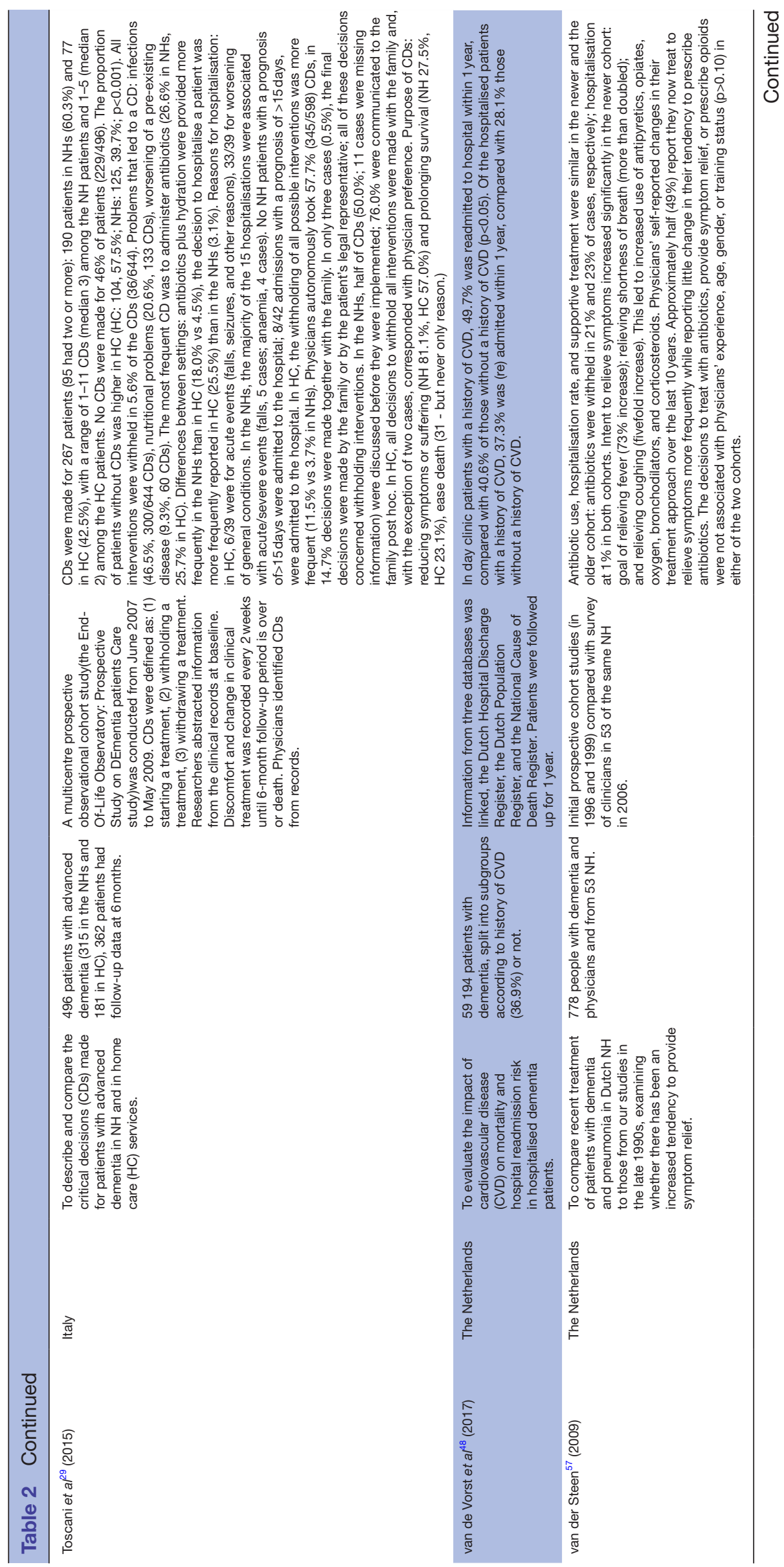




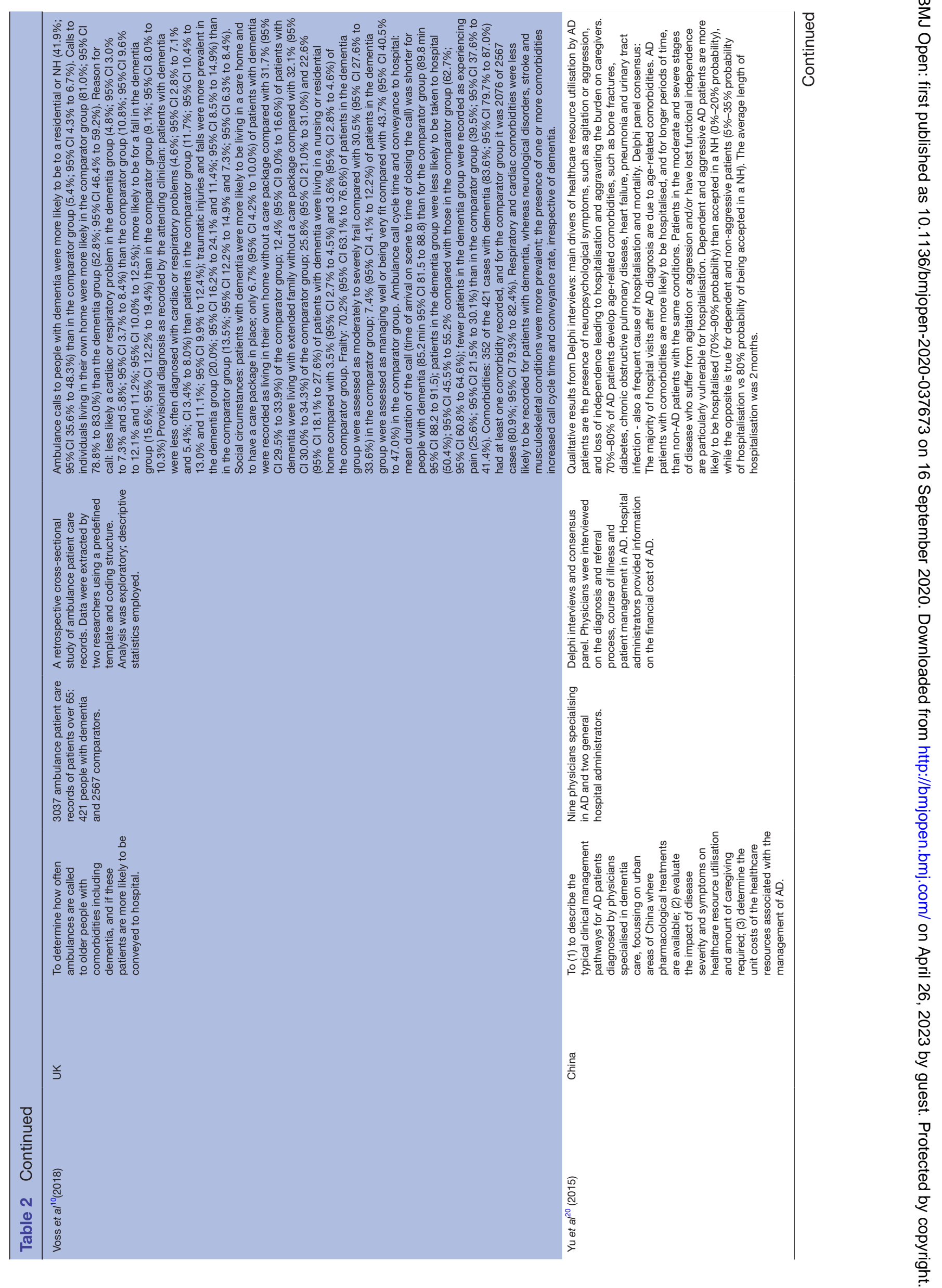




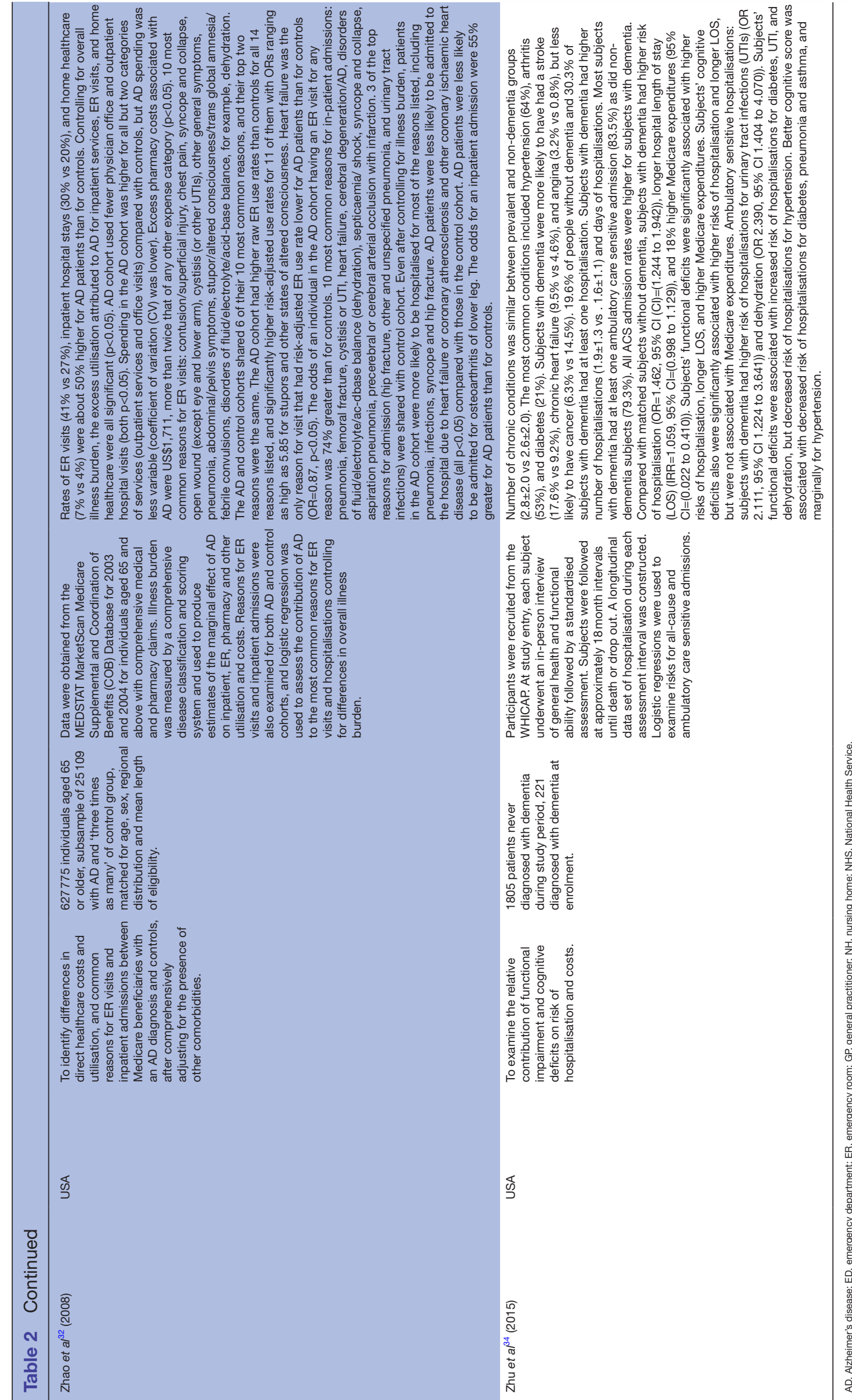


Table 3 Factors associated with urgent care use

\begin{tabular}{|c|c|c|}
\hline Factor & Increased urgent care use(*) & Decreased urgent care use $(t)$ \\
\hline More/specific comorbidities & 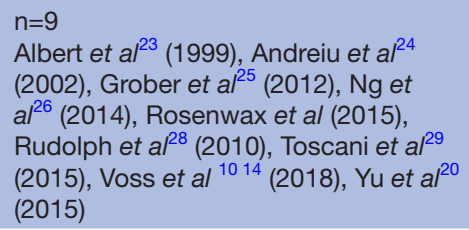 & \\
\hline $\begin{array}{l}\text { Having dementia (compared with } \\
\text { people without dementia) }\end{array}$ & $\begin{array}{l}\mathrm{n}=4 \\
\text { Husaini et }\left.a\right|^{33}(2003) \text {, Yu et } a l^{20}(2015) \text {, } \\
\text { Zhao et } a l^{32}(2008), \text { Zhu et } a^{34}(2015)\end{array}$ & $\begin{array}{l}\mathrm{n}=4 \\
\text { Albert et al }{ }^{23}(1999), \text { Rosenwax et } \\
\mathrm{al}^{27}(2015), \text { Voss et } a l^{10}{ }^{14}(2018), \\
\text { Zhao }^{32} \text { et al (2008) }\end{array}$ \\
\hline Presence of family carer & $\begin{array}{l}\mathrm{n}=4 \\
\text { Cogen et al }{ }^{40}(1992), \text { Ledgerd et al }{ }^{38} \\
(2016), \text { Ng et al } \\
\text { al }{ }^{27}(2014), \text { Rosenwax et }\end{array}$ & 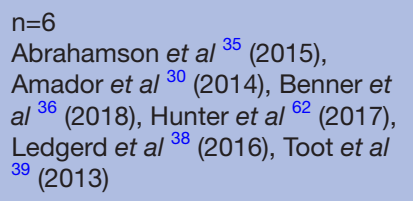 \\
\hline Older age & $\begin{array}{l}\mathrm{n}=5 \\
\text { Amador et al }{ }^{30}(2014), \text { Givens et al }{ }^{43} \\
2012, \text { Molloy et al }{ }^{44}(1991), \text { Rosenwax } \\
\text { et al }^{27} \text { (2015), Rudolph et al }{ }^{28} \text { (2010) }\end{array}$ & \\
\hline Behavioural Symptoms & $\begin{array}{l}\mathrm{n}=5 \\
\text { Andrieu et } a l^{24}(2002), \text { Goodman et al } \\
45(2017), \text { Ledgerd et al }{ }^{38}(2016) \text {, Toot } \\
\text { et al }{ }^{39}(2013) \text {, Yu et } a l^{20}(2015)\end{array}$ & \\
\hline Advance care planning & & 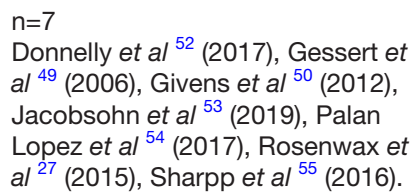 \\
\hline
\end{tabular}

\begin{tabular}{|c|c|c|c|}
\hline Ethnicity & $\begin{array}{l}\mathrm{n}=3 \\
\text { Agyemang et al }{ }^{48}(2016), \text { Gessert et al } \\
49 \text { (2006), Givens et al }{ }^{50}(2012)\end{array}$ & & $\begin{array}{l}\mathrm{n}=3 \\
\text { Albert et al }{ }^{23} \text { (1999), Husaini et al }{ }^{33} \\
\text { (2003), Rudolph et al }{ }^{28} \text { (2010) }\end{array}$ \\
\hline High dependency on others & $\begin{array}{l}\mathrm{n}=5 \\
\text { Andrieu et al }{ }^{24}(2002), \text { Cloutier et al }{ }^{42} \\
(2017), \text { Ledgerd et al }{ }^{38}(2016), \text { Ng et al } \\
{ }^{26} \text { (2014), Yu et al }{ }^{20}(2015)\end{array}$ & & $\begin{array}{l}\mathrm{n}=1 \\
\text { Grober et al }{ }^{25}(2012)\end{array}$ \\
\hline Geographical location of residence & $\begin{array}{l}\mathrm{n}=6 \\
\text { Gessert et al }{ }^{49}(2006), \text { Kim et al }{ }^{41} \\
(2019), \text { Molloy et al }{ }^{44}(1991), \mathrm{Ng} \text { et al } \\
{ }^{26}(2014), \text { Rosenwax et al }{ }^{27} \text { (2015), van } \\
\text { der Steen et al }{ }^{57} \text { (2009) }\end{array}$ & & \\
\hline Living in care facility & $\begin{array}{l}\mathrm{n}=3 \\
\text { Kim et al }{ }^{41} \text { (2019), Rosenwax et al }{ }^{27} \\
(2015), \text { Toscani et al }{ }^{29}(2015)\end{array}$ & $\begin{array}{l}\mathrm{n}=2 \\
\left(\text { Cloutier et al }{ }^{42}(2017) \text {, Toscani et }\right. \\
\left.\text { al }^{29}(2015)\right)\end{array}$ & \\
\hline Adequate outpatient services & & $\begin{array}{l}\mathrm{n}=4 \\
\text { Jacobsohn et al }{ }^{53} \text { (2019), Ledgerd } \\
\text { et al }{ }^{38}(2016), \text { Sadak et al }{ }^{56} \\
(2017), \text { Toot et al }{ }^{39}(2013)\end{array}$ & \\
\hline Gender & $\begin{array}{l}\mathrm{n}=4 \\
\text { Givens et al }{ }^{50}(2012), \text { Kim et al }{ }^{41} \\
\text { (2019), Rosenwax et al }{ }^{27}(2015) \text {, } \\
\text { Rudolph et al }{ }^{28}(2010)\end{array}$ & & \\
\hline $\begin{array}{l}\text { Higher levels of cognitive } \\
\text { impairment }\end{array}$ & $\begin{array}{l}\mathrm{n}=3 \\
\text { Albert et al }{ }^{23} \text { (1999), Molloy et al }{ }^{44} \\
\text { (1991), Zhu et al }{ }^{34} \text { (2015) }\end{array}$ & & $\begin{array}{l}\mathrm{n}=1 \\
\text { Rudolph et al }{ }^{28}(2010)\end{array}$ \\
\hline Technological support at home & & $\begin{array}{l}\mathrm{n}=3 \\
\text { Carter and Porrell et al }{ }^{21}(2005), \\
\text { Ledgerd et al }{ }^{38}(2016), \text { Toot et al } \\
{ }^{39}(2013)\end{array}$ & \\
\hline
\end{tabular}




\begin{tabular}{|c|c|c|c|}
\hline Factor & Increased urgent care use( $\left(^{\star}\right)$ & Decreased urgent care use(t) & No association with urgent care use \\
\hline Lower level of education & $\begin{array}{l}\mathrm{n}=2 \\
\text { Albert et al }{ }^{23} \text { (1999), Andrieu et al }{ }^{24} \\
(2002)\end{array}$ & & $\begin{array}{l}\mathrm{n}=1 \\
\text { Rudolph et al }{ }^{28} \text { (2010) }\end{array}$ \\
\hline Shorter duration of diagnosis & $\begin{array}{l}\mathrm{n}=2 \\
\text { Orrell and Bebbington }{ }^{51} \text { (1995), } \\
\text { Rudolph et } \mathrm{al}^{28} \text { (2010) }\end{array}$ & & \\
\hline Increase in nursing home staffing & & $\begin{array}{l}\mathrm{n}=2 \\
\text { Carter and Porrell et al }{ }^{21} \text { (2005), } \\
\text { Goodman et al }{ }^{45}(2017)\end{array}$ & \\
\hline
\end{tabular}

*Factors shown to increase urgent care use in one study only: low weight, life changes, more contacts with GP, humidity, ethical concerns about patient, legal concerns.

†Factors shown to prevent urgent care use in one study only: younger clinician age, Medicaid, the weekend, non profit status of care home.

$\ddagger$ Additional factors shown by one study to have no association with urgent care use: gender, income, marital status, proximity to emergency department. GP, general practitioner.

studies that found no association between comorbidities and accessing urgent care. ${ }^{30} 31$

Diagnosis of dementia

Twelve studies compared hospitalisation or emergency department use in dementia with those without dementia. Studies were evenly split between whether having dementia increased ${ }^{20}$ 32-34 $^{\text {or }}$ decreased ${ }^{10232732}$ urgent care use. Zhao et al found that people with dementia were more likely to be hospitalised overall than people without dementia, but they were less likely to be hospitalised for osteoarthritis or heart conditions. ${ }^{32}$ Two studies found no increase or decrease in hospital use for dementia compared with people without dementia. ${ }^{25} 27$

Role of informal and professional carers

Informal carers, in making decisions and providing support for people with dementia, were reported to prevent situations arising such that urgent care was needed..$^{30-35}$ However, a relationship strain or abuse or neglect from informal carers could cause increased

Table 4 Reasons for accessing urgent care

\begin{tabular}{|c|c|}
\hline Condition & References \\
\hline Infections & $\begin{array}{l}\mathrm{n}=18 \\
\left.\text { Amador et al }{ }^{30}(2014) \text { Benner et al }{ }^{36} \text { Carter and Porell }{ }^{21}(2005) \text {, Chang et al (2015) }\right)^{47} \text {, Clay }{ }^{69}(2008) \text {, D'Agata } \\
\text { et al }{ }^{60}(2013) \text {, Givens et al }{ }^{50}(2012) \text {, Givens et al }{ }^{43}(2015) \text {, Ledgerd et al }{ }^{38}(2016) \text {, Lin et al }{ }^{22}(2017) \text {, Mitchell } \\
\text { et al (2009), }{ }^{68} \text { Mitchell et al }{ }^{58}(2014) \text {, Rudolph et al }{ }^{28}(2010) \text {, Sharpp et al }{ }^{55}(2016) \text {, Sloane et al }{ }^{31}(2017) \text {, Toot } \\
\text { et al }{ }^{39} \text { (2013), Toscani et al }{ }^{39} \text { (2015), Zhao et al }{ }^{32} \text { (2008). }\end{array}$ \\
\hline $\begin{array}{l}\text { Behavioural symptoms and/or } \\
\text { delirium }\end{array}$ & $\begin{array}{l}\mathrm{n}=14 \\
\text { Andrieu et } a l^{24}(2002) \text {, Benner et al }{ }^{36}(2018) \text {, Boltz et al }{ }^{46}(2018) \text {, Chang et al }{ }^{47}(2015), \text { Givens et al }{ }^{50}(2012) \\
\text { Ledgerd et al }{ }^{38}(2016) \text {, Mitchell et al }{ }^{68}(2009) \text {, Rudolph et al }{ }^{28}(2010) \text {, Sadak et al }{ }^{56}(2017) \text {, Sharpp et al }{ }^{55} \\
\text { (2016), Sloane et al }{ }^{31}(2017) \text {, Toot et al }{ }^{39}(2013) \text {, Toscani et al }{ }^{29}(2015) \text {, Zhao et al }{ }^{32}(2008)\end{array}$ \\
\hline Falls/Injuries & $\begin{array}{l}\mathrm{n}=14 \\
\text { Amador et al }{ }^{30}(2014) \text {, Andrieu et al }{ }^{24}(2002) \text {, Benner et al }{ }^{36}(2018) \text {, Chang et al }{ }^{47}(2015) \text {, Givens et al }{ }^{50} \\
\text { (2012), Kim et al }{ }^{41}(2019) \text {, Ledgerd et al }{ }^{38}(2016) \text {, Lin et al }{ }^{22}(2017) \text {, Mitchell et al }{ }^{58}(2009) \text {, Rudolph et al }{ }^{28} \\
\text { (2010), Sadak et al }{ }^{56}(2017) \text {, Sharpp et al }{ }^{55}(2017) \text {, Sloane et } a l^{31}(2017) \text {, Zhao et al }{ }^{32} \text { (2008) }\end{array}$ \\
\hline Heart and/or respiratory disease & $\begin{array}{l}\mathrm{n}=11 \\
\text { Amador et al }{ }^{30}(2014) \text {, Chang et al }{ }^{47}(2015) \text {, Gviens et al }{ }^{50}(2012) \text {, Lin et al }{ }^{22} \text { (2017), Mitchell et al }{ }^{68}(2009) \text {, } \\
\text { Rudolph et al }{ }^{28}(2010) \text {, Sadak et al }{ }^{56}(2017) \text {, Sharpp et al }{ }^{55}(2016) \text {, Sloane et al }{ }^{31}(2017) \text {, van de Vorst et al }{ }^{48} \\
\text { (2017), Zhao et al }{ }^{32} \text { (2008) }\end{array}$ \\
\hline Syncope and/or seizures & $\begin{array}{l}\mathrm{n}=5 \\
\text { Mitchell et al }{ }^{68} \text { (2009), Rudolph et al }{ }^{28} \text { (2010), Sharpp et al }{ }^{55} \text { (2016), Sloane et al }{ }^{31} \text { (2017), Zhao et al }{ }^{32} \\
(2008)\end{array}$ \\
\hline Disability and/or immobility & $\begin{array}{l}n=3 \\
\text { Kim et al }{ }^{41} \text { (2019), Ledgerd et al }{ }^{38} \text { (2016), Sloane et al }{ }^{31}(2017)\end{array}$ \\
\hline Stroke & Chang et al ${ }^{47}$ (2015) \\
\hline Aphasia and/or dysphagia & Tian et al (2013) ${ }^{84}$ \\
\hline Incontinence & Ledgerd et al ${ }^{38}$ (2016) \\
\hline
\end{tabular}


urgent care use, and informal carers were also reported to increase use when the person with dementia is closer to death. ${ }^{26273840}$ Cogen et alfound that male carers increased urgent care use but there was no association with carer age or education level and urgent care use. ${ }^{40}$ The person with dementia living in a care facility was shown to increase access to urgent care, ${ }^{27} 2941$ although Toscani et al found that while there were more urgent situations with people with dementia living in care homes, they were less likely to go to hospital. ${ }^{29}$ Cloutier et al also found a decrease in hospitalisation after people with dementia were admitted to residential care. ${ }^{42}$

Symptoms and characteristics of the person with dementia Older age $\mathrm{e}^{2728304344}$ and the presence of behavioural symptoms ${ }^{2024383945}$ were reported to increase urgent care use. While there were no studies that reported these factors as being associated with a decrease in urgent care use, there were four ${ }^{23254647}$ and two ${ }^{2631}$ studies, respectively, that found no association. This was also the case with ethnicity; studies reported increased urgent care use for non-white compared with white people with dementia, ${ }^{48-50}$ while others reported no association. ${ }^{23} 2833$ Similarly, higher dependency, ${ }^{20} 24263842$ higher level of cognitive impairment $^{233444}$ and lower levels of education ${ }^{2324}$ were shown by some studies to be associated with increased urgent care use, while others showed no association. ${ }^{25} 28$ There was some association with gender and increased urgent care use, with males reported as more likely to be hospitalised or visit emergency departments ${ }^{27} 280$ and one study reporting females more likely to have hip fractures. ${ }^{41}$

There were some differences in reasons for urgent care use according to type of dementia, with Chang et al showing that people with vascular dementia had higher frequencies of falls and delirium, and Rosenwax et al reporting fewer visits to emergency departments for people with Alzheimer's disease. ${ }^{27} 47$ Chang et al found no difference in recurrent hospitalisations between dementia type. ${ }^{47}$ Two studies found that a shorter duration of dementia diagnosis was specifically associated with emergency psychiatric care. $^{2851}$

\section{Variation in service organisation and location}

Advance care planning was cited as preventative for urgent care use, but identified as difficult to put in place. $^{27495052-55}$ Adequate outpatient services, specifically care coordinators and support in the home, were also identified to prevent urgent care use. ${ }^{38395356}$ Accidents in the home were highlighted as key causes of crises and resulting access to urgent care, and technological support within the home was seen as a way to avoid this. ${ }^{2138} 39$

Six studies reported associations between geographical locations and increased urgent care use, including different levels of urgent care use in different countries, ${ }^{445}$ more urgent care use in rural over urban areas $^{274149}$ and more urgent care use in northern over southern regions. ${ }^{26}$

\section{Stakeholder experiences of urgent care use in dementia}

Thirty-one studies considered stakeholder experiences in more detail. Stakeholders experienced various situations in urgent care: from hospitalisation decisions, ${ }^{35} 3754$ to withholding treatment at the end of life, ${ }^{29} 375859$ to prescribing antibiotics. ${ }^{575860}$

Three factors were considered to play particularly important roles in how people with dementia access urgent care and how care is experienced.

Knowledge of dementia as a condition and individual patient back stories

Specialist and in-depth knowledge of both dementia as a condition, and each individual person with dementia, was a priority in 20 studies. People with dementia were commonly not involved in decision making. ${ }^{461}$ They were reported to be in less pain and more likely to be marked as less urgent than those without dementia. ${ }^{1027}$ There was concern among carers that a dementia diagnosis led to exclusion from specialist care services that a diagnosis of cancer might not. ${ }^{42} 45$ General practitioners (GPs) were reported to find behavioural symptoms in care homes as the most challenging part of their job, ${ }^{45}$ with patients with dementia seen as a burden on time. ${ }^{5362}$

A lack of understanding of dementia was identified as a trigger for crises for people with dementia across settings. Increased training in dementia was reported as important to prevent crises in care homes, ${ }^{384563}$ but specific staff employed as dementia specialists were reported as having a particularly beneficial effect. These could be placed within care homes ${ }^{39} 450$ or based in outpatient, primary care settings with constant contact with care homes. ${ }^{38} 453$

Emergency department staff had specific challenges related to the time-pressured environment, where the focus was on the acute presenting problem rather than dementia. ${ }^{53626465}$ This could be compounded by the presence of delirium. ${ }^{64}$ Dementia could also make it harder for staff to identify the reason for visiting the emergency department, which lead to undertriaging and longer waiting times for patients. ${ }^{65}$ That some people with dementia were unable to describe their symptoms was a particular problem. ${ }^{596265}$ For those in care homes, information exchange between informal carers, professionals and emergency services was seen as a pivotal factor to improve access to urgent care, and one which was often lacking. ${ }^{41} 4554555866$

Inadequate health and social care support leads to accessing urgent care

Fourteen studies reported that outpatient care and home support were not sufficient for people with dementia. There were two sides to this issue. First, a lack of outpatient or community support would cause crises, with situations worsening for people with dementia and their caregivers until hospital admission is necessary. ${ }^{456} 53$ Second, informal carers find themselves in a challenging situation and have nowhere to go for support other than an emergency department, despite acknowledging 
that the situation may not be urgent. ${ }^{38} 5267$ Once a crisis point had been reached, support services became available where they were not available before. ${ }^{67}$ This could be due to service organisation factors, for example, not being able to access a psychiatrist unless the person with dementia is admitted, ${ }^{45}$ or economic factors, with insufficient funds and staffing in outpatient services. ${ }^{52}$

Professional carers reported feeling frustrated by limitations on their ability to avoid emergency situations in dementia. ${ }^{55}$ There were not always the staff available to control extreme behavioural symptoms. ${ }^{45}$ Additionally, professional carers report resistance from informal carers to engage in end-of-life discussions, resulting in people with dementia being hospitalised or aggressively treated even in late stages of their condition. ${ }^{54}$

\section{Influence of informal carers in accessing urgent care}

The role of the informal carer was explored in detail in 12 studies. Caregiver burden, characterised by negative emotional state, strain and sleep disturbance, was seen as a crucial factor in why people with dementia end up accessing urgent care, particularly emergency departments and hospitals. ${ }^{39535662}$ Caregiver strain was found to be associated with a change in functioning of people with dementia prior to hospital admission. ${ }^{46}$ Sudden caregiver physical illness or limitations on providing care also lead to urgent situations arising for people with dementia. ${ }^{38} 3956$ This results in a vicious circle, with stress and guilt causing further damage to caregiver well-being. ${ }^{56}$ Correspondingly, support for carers was critical for preventing urgent situations in dementia, with strategies to maintain selfcare and access to respite, including emergency respite in the home, identified as paramount. ${ }^{383956}$

Another challenge of informal caring was the need to identify symptoms or avoid situations that may lead to urgent care situations. ${ }^{56}$ Carers' lack of knowledge about how to recognise and manage healthcare situations, and what the treatment options were, lead to increased urgent care use. ${ }^{53}$ Additionally, as the situation escalates, caregivers may be under pressure to make decisions quickly, leading to inappropriate care ${ }^{56}$ Caregiver education and counselling was therefore reported to be key in reducing urgent care use and providing support for caregivers to manage changes in symptoms appropriately. ${ }^{38} 39$ This was seen as particularly important regarding end of life decision making in urgent situations, for example Mitchell et al found that carers who had been counselled about expected complications and prognosis in advanced dementia were less likely to choose burdensome interventions such as hospitalisation in the last 3 months of life. ${ }^{68}$

Barriers between informal carers and health and social care services was a particular challenge that lead to urgent care use. Lack of support meant that hospitalisation resulting in the person with dementia discharged to residential care was experienced as a relief for stressed carers, who had felt guilty about making this decision on their own. ${ }^{52}$ Carers reported finding it hard to speak to GPs and community services about potential crisis situations. ${ }^{53}$ There were also barriers identified by professionals, with reports from care facility and emergency department staff of carers directly contradicting instructions in advance care plans, ordering more burdensome interventions. $^{35} 5969$ Medical professionals were seen to take the lead in decision making in urgent care. ${ }^{29} 37$ Involvement of informal carers was reported to lead to more aggressive treatment, for example, carer awareness of infection episodes for people with dementia in care homes lead to increased hospital transfers. ${ }^{43}$ Informal carers were not always informed by care home staff or clinicians if their relative with dementia had an infection, with under half being told if the patient with dementia had been prescribed antibiotics. ${ }^{58}$

\section{DISCUSSION}

The existing research exploring urgent care in dementia reports complex care scenarios affected by comorbidities, characteristics associated with dementia as a condition such as memory loss and behavioural symptoms, informal and professional carers, and the quality of outpatient healthcare services. A lack of understanding of dementia and knowledge about the patient as an individual, inadequate community support, and competing demands of informal and professional carers can cause additional challenges to the person with dementia receiving appropriate care.

The strengths of this scoping review are the systematic nature of the search and data extraction process. The broad research question and inclusion criteria allowed a wide variety of studies to be included. The involvement of people with dementia and informal carers in the design of the study and throughout the review process strengthened the review in ensuring the research addressed the concerns of key stakeholders. The primary limitation was the inclusion of only English language research. Additionally, the included studies explored dementia as an overarching condition, and therefore, the different effects of different types of dementia were not examined. Scoping review methodology does not include a formal quality appraisal process, which means the scientific quality of the studies has not been taken into account.

Considering the nature of a scoping review and the broad research questions, it was unsurprising that the 54 included studies explored a broad spectrum of factors associated with urgent situations for people with dementia, and that there were some competing findings. Sociodemographic factors such as older age, gender, ethnicity, lower education and geographical location were shown by some studies to increase urgent care use, while other studies showed no association. However, the studies did not discuss whether these factors are dementia specific, and there is research showing similar patterns in other patient groups. ${ }^{70-72}$ Similarly, comorbidity increases acute care use in other chronic illnesses. ${ }^{73}$ The heterogeneity of dementia, both in terms of manifest symptoms and disease severity, adds to the challenge of unpicking 
the relationship between a dementia diagnosis and urgent care use. That very few of the studies differentiated between dementia type meant that this could not be unpicked in this review.

There are two key implications for policy-makers and clinicians arising from this review. However, both rely on an increase of support for people with dementia and their carers in the community. First, a large proportion of the included studies identified insufficient care and support, in a variety of areas, as a key cause for crises occurring, as well as the reason people with dementia are admitted to hospital unnecessarily. Economic causes, such as low staffing levels in care homes or lack of services, were partly to blame, which is also reflected in other literature. $^{74}$ However, a lack of communication between existing services was also detrimental to appropriate care, which has also been identified in other studies of dementia services. ${ }^{4}$ There is much recent research highlighting the benefits of integrated care for people with dementia, especially regarding patient-specific information being easily shared across different services, both between health and social care services and between community and hospital based services. ${ }^{74}$ The use of dementia specialists has also been highlighted as key to higher quality care, which was also identified in the review. ${ }^{76}$ The results of this review add to this, with a specific focus on how integrated services can both prevent inappropriate urgent care use and improve experiences.

The second key implication is reflected in the different priorities of stakeholders, which were particularly prevalent when it came to end-of-life decisions that arise in urgent situations. Advance care planning was identified as crucial in preventing unnecessary hospitalisation in urgent situations, but can be a challenge to implement, as has been discussed in the wider end-of-life care literature. ${ }^{77}$ Timely planning on what to do in urgent situations is advised to avoid problems of decision-making capacity, ${ }^{78}$ but memory clinics and GPs have highlighted challenges of initiating these conversations when people are adjusting to living with their new diagnosis and capacity is not yet an issue. ${ }^{790}$ Education of caregivers about how dementia progresses and the situations that may arise at end of life was highlighted as key in this review, as it has been in others. ${ }^{78}$ However, this will again rely on more resources and support for people with dementia and their families prior to urgent care situations arising.

Future research in this area will benefit from inclusion of the perspectives of people with dementia, which were included in only 6 of the 54 studies. ${ }^{38} 3945466566$ This may be due to the complex nature of urgent care situations, where time constraints make more dementia-appropriate recruitment methods ${ }^{81}$ challenging, and those in advanced dementia not being able to participate in interviews. Future research could adapt from more traditional methodologies to explore the experiences of people with dementia. For example, studies have successfully used ethnographic or observational techniques to capture how decisions are made in non-urgent care settings, and using similar methods in urgent care would be beneficial in providing guidance and training to professionals. ${ }^{62}$ The included studies are dominated by large cohort studies, providing descriptive data on how many people with dementia in different contexts and settings access different types of urgent care, and what characteristics are associated with this. A future review could provide a meta-analysis of the available cohort studies in this area, in order to provide a definitive list of factors that increase or prevent urgent care use.

Twitter Jemima Dooley @DrMimaDooley and Matthew Booker @MatthewBooker

Contributors JD came up with the concept of the review, ran the patient and public involvement groups, lead on every stage of the review process and writing the article. $\mathrm{RB}$ and $\mathrm{MB}$ helped with protocol development and providing comments on the article. PX helped with protocol development, the screening and extraction process, and providing comments on the article.

Funding This project is funded by the National Institute for Health Research (NIHR) School for Primary Care Research (project reference 74370).

Disclaimer The views expressed are those of the author and not necessarily those of the NIHR or the Department of Health and Social Care.

Competing interests None declared.

Patient and public involvement Patients and/or the public were involved in the design, or conduct, or reporting, or dissemination plans of this research. Refer to the Methods section for further details.

Patient consent for publication Not required.

Provenance and peer review Not commissioned; externally peer reviewed.

Data availability statement No data are available. N/A.

Open access This is an open access article distributed in accordance with the Creative Commons Attribution Non Commercial (CC BY-NC 4.0) license, which permits others to distribute, remix, adapt, build upon this work non-commercially, and license their derivative works on different terms, provided the original work is properly cited, appropriate credit is given, any changes made indicated, and the use is non-commercial. See: http://creativecommons.org/licenses/by-nc/4.0/.

ORCID iDs

Jemima Dooley http://orcid.org/0000-0003-3418-8112

Penny Xanthopoulou http://orcid.org/0000-0002-1510-3382

\section{REFERENCES}

1 Jacomb PA, Jorm AF. Personality change in dementia of the Alzheimer type. Int J Geriatr Psychiatry 1996;11:201-7.

2 Poblador-Plou B, Calderón-Larrañaga A, Marta-Moreno J, et al. Comorbidity of dementia: a cross-sectional study of primary care older patients. BMC Psychiatry 2014;14:84.

3 Browne J, Edwards DA, Rhodes KM, et al. Association of comorbidity and health service usage among patients with dementia in the UK: a population-based study. BMJ Open 2017;7:e012546.

4 Martin A, O'Connor S, Jackson C. A scoping review of gaps and priorities in dementia care in Europe. Dementia 2018:1471301218816250

5 Hamann J, Bronner K, Margull J, et al. Patient participation in medical and social decisions in Alzheimer's disease. J Am Geriatr Soc 2011;59:2045-52.

6 Smebye KL, Kirkevold M, Engedal K. How do persons with dementia participate in decision making related to health and daily care? A multi-case study. BMC Health Serv Res 2012;12:241.

7 Dooley J, Bass N, Livingston G, et al. Involving patients with dementia in decisions to initiate treatment: effect on patient acceptance, satisfaction and medication prescription. Br J Psychiatry 2019;214:213-7.

8 Voss S, Black S, Brandling J, et al. Home or hospital for people with dementia and one or more other multimorbidities: what is the potential to reduce avoidable emergency admissions? the HOMEWARD project protocol. BMJ Open 2017;7:4.

9 Lakey L. Counting the cost: Alzheimer's society, 2009. 
10 Voss S, Brandling J, Taylor H, et al. How do people with dementia use the ambulance service? A retrospective study in England: the HOMEWARD project. BMJ Open 2018;8:e022549.

11 Livingston G, Leavey G, Manela M, et al. Making decisions for people with dementia who lack capacity: qualitative study of family carers in UK. BMJ 2010;341:c4184.

12 Booker MJ, Shaw ARG, Purdy S, et al. 'Primary care sensitive' situations that result in an ambulance attendance: a conversation analytic study of UK emergency '999' call recordings. BMJ Open 2018;8:e023727.

13 Alzheimer Europe Reports. Alzheimer Europe survey: who cares?The state of dementia care in Europe: Alzeimer Europe, 2006.

14 Voss S, Brandling J, Black S, et al. Carer and clinician perceptions of the use of emergency medical services by people with dementia: a qualitative study. Prim Health Care Res Dev 2018:1-4.

15 NHS England. About urgent and emergency care. Available: https:// www.england.nhs.uk/urgent-emergency-care/about-uec/

16 Munn Z, Peters MDJ, Stern C, et al. Systematic review or scoping review? guidance for authors when choosing between a systematic or scoping review approach. BMC Med Res Methodol 2018;18:143.

17 Peters MDJ, Godfrey CM, Khalil H, et al. Guidance for conducting systematic scoping reviews. Int J Evid Based Healthc 2015;13:141-6.

18 Kreis J, Puhan MA, Schünemann HJ, et al. Consumer involvement in systematic reviews of comparative effectiveness research. Health Expect 2013;16:323-37.

19 NIHR. Does health and social care provision for the community dwelling older population help to reduce unplanned secondary care, support timely appropriate discharge, and improve patient wellbeing? a mixed method meta review of systematic reviews. PROSPERO, 2018. Available: https://www.crd.york.ac.uk/ PROSPERO/display_record.php?RecordID=87534 [Accessed 28 Nov 19].

$20 \mathrm{Yu}$ X, Chen S, Chen X, et al. Clinical management and associated costs for moderate and severe Alzheimer's disease in urban China: a Delphi panel study. Trans/ Neurodegener 2015;4:15.

21 Carter MW, Porell FW. Vulnerable populations at risk of potentially avoidable hospitalizations: the case of nursing home residents with Alzheimer's disease. Am J Alzheimers Dis Other Demen 2005;20:349-58.

22 Lin P-J, Zhong Y, Fillit HM, et al. Hospitalizations for ambulatory care sensitive conditions and unplanned readmissions among Medicare beneficiaries with Alzheimer's disease. Alzheimers Dement 2017;13:1174-8.

23 Albert SM, Costa R, Merchant C, et al. Hospitalization and Alzheimer's disease: results from a community-based study. $J$ Gerontol A Biol Sci Med Sci 1999;54A:M267-71.

24 Andrieu S, Reynish E, Nourhashemi F, et al. Predictive factors of acute hospitalization in 134 patients with Alzheimer's disease: a one year prospective study. Int J Geriatr Psychiatry 2002;17:422-6.

25 Grober E, Sanders A, Hall CB, et al. Very mild dementia and medical comorbidity independently predict health care use in the elderly. $J$ Prim Care Community Health 2012;3:23-8.

$26 \mathrm{Ng} \mathrm{S}$, Morgan RO, Walder A, et al. Functional decline predicts emergency department use in veterans with dementia. Am J Alzheimers Dis Other Demen 2014;29:362-71.

27 Rosenwax L, Spilsbury K, Arendts G, et al. Community-Based palliative care is associated with reduced emergency department use by people with dementia in their last year of life: a retrospective cohort study. Palliat Med 2015;29:727-36.

28 Rudolph JL, Zanin NM, Jones RN, et al. Hospitalization in community-dwelling persons with Alzheimer's disease: frequency and causes. J Am Geriatr Soc 2010;58:1542-8.

29 Toscani F, van der Steen JT, Finetti S, et al. Critical decisions for older people with advanced dementia: a prospective study in long-term institutions and district home care. J Am Med Dir Assoc 2015;16:535. e13-535.e20.

30 Amador S, Goodman C, King D, et al. Emergency ambulance service involvement with residential care homes in the support of older people with dementia: an observational study. BMC Geriatr 2014;14:95-101.

31 Sloane PD, Schifeling $\mathrm{CH}$, Beeber AS, et al. New or worsening symptoms and signs in community-dwelling persons with dementia: incidence and relation to use of acute medical services. J Am Geriatr Soc 2017;65:808-14.

32 Zhao Y, Kuo T-C, Weir S, et al. Healthcare costs and utilization for Medicare beneficiaries with Alzheimer's. BMC Health Serv Res 2008;8:108.

33 Husaini BA, Sherkat DE, Moonis M, et al. Racial differences in the diagnosis of dementia and in its effects on the use and costs of health care services. Psychiatr Serv 2003;54:92-6.
34 Zhu CW, Cosentino S, Ornstein K, et al. Use and cost of hospitalization in dementia: longitudinal results from a communitybased study. Int J Geriatr Psychiatry 2015;30:833-41.

35 Abrahamson K, Bernard B, Magnabosco L, et al. The experiences of family members in the nursing home to hospital transfer decision. BMC Geriatr 2016;16:184.

36 Benner M, Steiner V, Pierce LL. Family caregivers' reports of hospitalizations and emergency department visits in communitydwelling individuals with dementia. Dementia 2018;17:585-95.

37 Lacey D. Tube feeding, antibiotics, and hospitalization of nursing home residents with end-stage dementia: perceptions of key medical decision-makers. Am J Alzheimers Dis Other Demen 2005;20:211-9.

38 Ledgerd R, Hoe J, Hoare Z, et al. Identifying the causes, prevention and management of crises in dementia. An online survey of stakeholders. Int J Geriatr Psychiatry 2016;31:638-47.

39 Toot S, Hoe J, Ledgerd R, et al. Causes of crises and appropriate interventions: the views of people with dementia, carers and healthcare professionals. Aging Ment Health 2013;17:328-35

40 Cogen R, Patterson B, Chavin S, et al. Surrogate decision-maker preferences for medical care of severely demented nursing home patients. Arch Intern Med 1992;152:1885-8.

$41 \mathrm{Kim}$ J, Choi Y, Park E-C. Incidence of hip fracture among long-term care insurance beneficiaries with dementia: comparison of home care and institutional care services. BMC Geriatr 2019;19:152.

42 Cloutier DS, Penning MJ. Janus at the crossroads: perspectives on long-term care trajectories for older women with dementia in a Canadian context. Gerontologist 2017;57:68-81.

43 Givens JL, Spinella S, Ankuda CK, et al. Healthcare proxy awareness of suspected infections in nursing home residents with advanced dementia. J Am Geriatr Soc 2015;63:1084-90.

44 Molloy DW, Guyatt GH, Alemayehu E, et al. Factors affecting physicians' decisions on caring for an incompetent elderly patient: an international study. CMAJ 1991;145:947-61.

45 Goodman C, Davies SL, Gordon AL, et al. Optimal NHS service delivery to care homes: a realist evaluation of the features and mechanisms that support effective working for the continuing care of older people in residential settings. Southampton (UK): Health Services and Delivery Research, 2017.

46 Boltz M, Lee KH, Chippendale T, et al. Pre-Admission functional decline in hospitalized persons with dementia: the influence of family caregiver factors. Arch Gerontol Geriatr 2018;74:49-54.

47 Chang C-C, Lin P-H, Chang Y-T, et al. The impact of admission diagnosis on recurrent or frequent hospitalizations in 3 dementia subtypes: a hospital-based cohort in Taiwan with 4 years longitudinal Follow-Ups. Medicine 2015;94:e2091.

48 Agyemang $\mathrm{C}$, van de Vorst IE, Koek HL, et al. Ethnic variations in prognosis of patients with dementia: a prospective nationwide registry linkage study in the Netherlands. J Alzheimers Dis 2017;56:205-13.

49 Gessert CE, Haller IV, Kane RL, et al. Rural-Urban differences in medical care for nursing home residents with severe dementia at the end of life. J Am Geriatr Soc 2006;54:1199-205

50 Givens JL, Selby K, Goldfeld KS, et al. Hospital transfers of nursing home residents with advanced dementia. J Am Geriatr Soc 2012;60:905-9.

51 Orrell M, Bebbington P. Life events and senile dementia I. admission, deterioration and social environment change. Psychol Med 1995;25:373-86.

52 Donnelly N-A, Humphries N, Hickey A, et al. "We don't have the infrastructure to support them at home": How health system inadequacies impact on long-term care admissions of people with dementia. Health Policy 2017;121:1280-7.

53 Jacobsohn GC, Hollander M, Beck AP, et al. Factors influencing emergency care by persons with dementia: Stakeholder perceptions and unmet needs. J Am Geriatr Soc 2019;67:711-8.

54 Palan Lopez R, Mitchell SL, Givens JL. Preventing Burdensome transitions of nursing home residents with advanced dementia: it's more than advance directives. J Palliat Med 2017;20:1205-9.

55 Sharpp TJ, Young HM. Experiences of frequent visits to the emergency department by residents with dementia in assisted living Geriatr Nurs 2016;37:30-5.

56 Sadak T, Foster Zdon S, Ishado E, et al. Potentially preventable hospitalizations in dementia: family caregiver experiences. Int Psychogeriatr 2017;29:1201-11.

57 van der Steen JT, Meuleman-Peperkamp I, Ribbe MW. Trends in treatment of pneumonia among Dutch nursing home patients with dementia. J Palliat Med 2009;12:789-95.

58 Mitchell SL, Shaffer ML, Loeb MB, et al. Infection management and multidrug-resistant organisms in nursing home residents with advanced dementia. JAMA Intern Med 2014;174:1660-7. 
59 Smith AK, Fisher J, Schonberg MA, et al. Am I doing the right thing? provider perspectives on improving palliative care in the emergency department. Ann Emerg Med 2009;54:e1:86-93.

60 D'Agata E, Loeb MB, Mitchell SL. Challenges in assessing nursing home residents with advanced dementia for suspected urinary tract infections. J Am Geriatr Soc 2013;61:62-6.

61 Le Guen J, Boumendil A, Guidet B, et al. Are elderly patients opinions sought before admission to an intensive care unit? results of the ICE-CUB study. Age Ageing 2016;45:303-9.

62 Hunter KF, Parke B, Babb M, et al. Balancing safety and harm for older adults with dementia in rural emergency departments: healthcare professionals' perspectives. Rural Remote Health $2017 ; 17$.

63 Kupeli N, Leavey G, Harrington J, et al. What are the barriers to care integration for those at the advanced stages of dementia living in care homes in the UK? health care professional perspective. Dementia 2018;17:164-79.

64 LaMantia MA, Messina FC, Jhanji S, et al. Emergency medical service, nursing, and physician providers' perspectives on delirium identification and management. Dementia 2017;16:329-43.

65 Parke B, Hunter KF, Strain LA, et al. Facilitators and barriers to safe emergency department transitions for community dwelling older people with dementia and their caregivers: a social ecological study. Int J Nurs Stud 2013;50:1206-18.

66 Robinson CA, Bottorff JL, Lilly MB, et al. Stakeholder perspectives on transitions of nursing home residents to hospital emergency departments and back in two Canadian provinces. J Aging Stud 2012;26:419-27.

67 Peel E, Harding R. 'It's a huge maze, the system, it's a terrible maze': dementia carers' constructions of navigating health and social care services. Dementia 2014;13:642-61.

68 Mitchell SL, Teno JM, Kiely DK, et al. The clinical course of advanced dementia. N Engl J Med 2009;361:1529-38.

69 Clay MT. Factors leading to older people in care homes being admitted to hospital as emergencies (thesis): University of Surrey, 2008.

70 Stingone JA, Claudio L. Disparities in the use of urgent health care services among asthmatic children. Ann Allergy Asthma Immunol 2006;97:244-50.

71 Hong R, Baumann BM, Boudreaux ED. The emergency department for routine healthcare: race/ethnicity, socioeconomic status, and perceptual factors. J Emerg Med 2007;32:149-58.
72 Platts-Mills TF, Hunold KM, Weaver MA, et al. Pain treatment for older adults during prehospital emergency care: variations by patient gender and pain severity. J Pain 2013;14:966-74.

73 Hutchinson AF, Graco M, Rasekaba TM, et al. Relationship between health-related quality of life, comorbidities and acute health care utilisation, in adults with chronic conditions. Health Qual Life Outcomes 2015;13:69.

74 Lyketsos CG. Prevention of unnecessary hospitalization for patients with dementia: the role of ambulatory care. JAMA 2012;307:197-8.

75 Murphy AM, Shwartz JL. Innovative treatment models for alzheimer's disease and dementia care: Exploring an "aging in place" approach that maximizes cost savings and patient-caregiver wellbeing. $J$ Health Care Finance 2018;44.

76 Backhouse A, Richards DA, McCabe R, et al. Stakeholders perspectives on the key components of community-based interventions coordinating care in dementia: a qualitative systematic review. BMC Health Serv Res 2017;17:767.

77 Gaster B, Larson EB, Curtis JR. Advance directives for dementia: meeting a unique challenge. JAMA 2017;318:2175-6.

78 Robinson L, Dickinson C, Rousseau N, et al. A systematic review of the effectiveness of advance care planning interventions for people with cognitive impairment and dementia. Age Ageing 2012;41:263-9.

79 Tilburgs B, Vernooij-Dassen M, Koopmans R, et al. Barriers and facilitators for GPs in dementia advance care planning: a systematic integrative review. PLoS One 2018;13:e0198535.

80 Bailey C, Dooley J, McCabe R. 'How do they want to know?' doctors' perspectives on making and communicating a diagnosis of dementia. Dementia 2019;18:3004-22.

81 Beattie E, Fielding E, O'Reilly M, et al. Recruitment of individuals with dementia and their carers for social research: lessons learned from nine studies. Res Gerontol Nurs 2018;11:119-28.

82 Dooley J, Bailey C, McCabe R. Communication in healthcare interactions in dementia: a systematic review of observational studies. Int Psychogeriatr 2015;27:1277-300.

83 Salib E, Sharp N. Does the weather influence dementia admissions? Int J Geriatr Psychiatry 1999;14:925-35.

84 Tian H, Abouzaid S, Sabbagh MN, et al. Health care utilization and costs among patients with AD with and without dysphagia. Alzheimer Dis Assoc Disord 2013;27:138-44. 